\title{
Linear space string correction algorithm using the Damerau-Levenshtein distance
}

\author{
Chunchun Zhao* (D) and Sartaj Sahni \\ From 8th IEEE International Conference on Computational Advances in Bio and medical Sciences (ICCABS 2018) \\ Las Vegas, NV, USA. 18-20 October 2018
}

\begin{abstract}
Background: The Damerau-Levenshtein (DL) distance metric has been widely used in the biological science. It tries to identify the similar region of DNA,RNA and protein sequences by transforming one sequence to the another using the substitution, insertion, deletion and transposition operations. Lowrance and Wagner have developed an $O(m n)$ time $O(m n)$ space algorithm to find the minimum cost edit sequence between strings of length $m$ and $n$, respectively. In our previous research, we have developed algorithms that run in $O(m n)$ time using only $O(s * \min \{m, n\}+m+n)$ space, where $s$ is the size of the alphabet comprising the strings, to compute the DL distance as well as the corresponding edit sequence. These are so far the fastest and most space efficient algorithms. In this paper, we focus on the development of algorithms whose asymptotic space complexity is linear.

Results: We develop linear space algorithms to compute the Damerau-Levenshtein (DL) distance between two strings and determine the optimal trace (corresponding edit operations.)

Extensive experiments conducted on three computational platforms-Xeon E5 2603, 17-x980 and Xeon E5 2695-show that, our algorithms, in addition to using less space, are much faster than earlier algorithms.

Conclusion: Besides using less space than the previously known algorithms,significant run-time improvement was seen for our new algorithms on all three of our experimental platforms. On all platforms, our linear-space cache-efficient algorithms reduced run time by as much as $56.4 \%$ and $57.4 \%$ in respect to compute the DL distance and an optimal edit sequences compared to previous algorithms. Our multi-core algorithms reduced the run time by up to $59.3 \%$ compared to the best previously known multi-core algorithms.
\end{abstract}

Keywords: Edit distance, Damerau-Levenshtein distance, Linear space, String correction

\footnotetext{
*Correspondence: zhaochunchun@gmail.com

Department of Computer and Information Science and Engineering,

University of Florida, Gainesville, 32611 FL, USA
}

\section{Background Introduction \\ The Damerau-Levenshtein (DL) distance between two strings is the minimum number of substitutions, inserts, deletes, and transpositions of adjacent characters required to transform one string into the other. Some of the appli- cations of the DL are spelling error correction [1-3], comparing packet traces [4], data mining and cluster- ing [5], quantifying the similarity of biological sequences, and gene function prediction [6], analysis of B cell recep- \\ tor repertoire data [7], virus detection in software [8],}

clustering of RNA-seq read se6gments [9], DNA repeats detection [10], and codes for DNA based storage [11]. In some of these applications (e.g., spelling error correction), the strings are rather small while in others (e.g., comparing protein sequences) the strings could be tens of thousands of characters long [12], and in yet others (e.g., comparing chromosomes) they could be millions of characters long [13].

Other string edit distances used in the literature permit only a proper subset of the operations permitted by the DL distance. For example, in the Levenshtein distance [14] transpositions are not permitted, in the Hamming distance [15] only substitutions are permitted, and in the 

$A: a_{1} a_{2} a_{3} \ldots a_{i-1}+a_{i} \quad A: a_{1} a_{2} a_{3} \ldots a_{i}$
B: $b_{1} b_{2} b_{3} \ldots . b_{j-1}+b_{j}$
$B: b_{1} b_{2} b_{3} \ldots b_{j-1}+b_{j}$

(a)

(b)
$A: a_{1} a_{2} a_{3} \ldots . a_{i-1}-a_{i}$
A: $a_{1} a_{2} a_{3} \ldots a_{k-1} a_{k} a_{k+1} \ldots a_{i}$
$B: b_{1} b_{2} b_{3} \ldots . b_{j}$
$B: b_{1} b_{2} b_{3} \ldots b_{1-1} b_{1} b_{1+1} \ldots b_{j}$

(c)

(d) translate $A[k: i]$ to $B[l: j]$

where $\left(a_{k}, b_{j}\right)$ and $\left(b_{1}, a_{i}\right)$ form a transposition opportunity.

Fig. $1 \mathrm{DL}$ distance recurrence. a substitution. b insertion. c deletion. $\mathbf{d}$ translate $A[k: i]$ to $B[l: j]$ where $\left(a_{k}, b_{j}\right)$ and $\left(b_{l}, a_{j}\right)$ form a transposition opportunity

\section{A: $d$ a $f$ a $c$ \\ $B: f d$ b b e c}

Fig. 2 DL trace example [18]

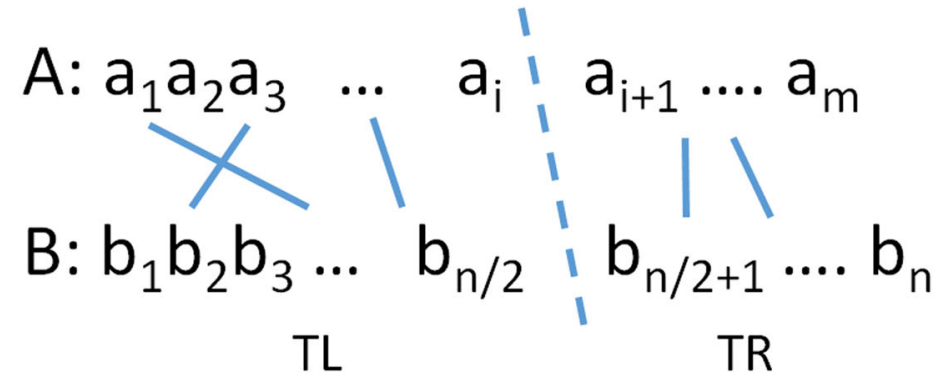

(a)

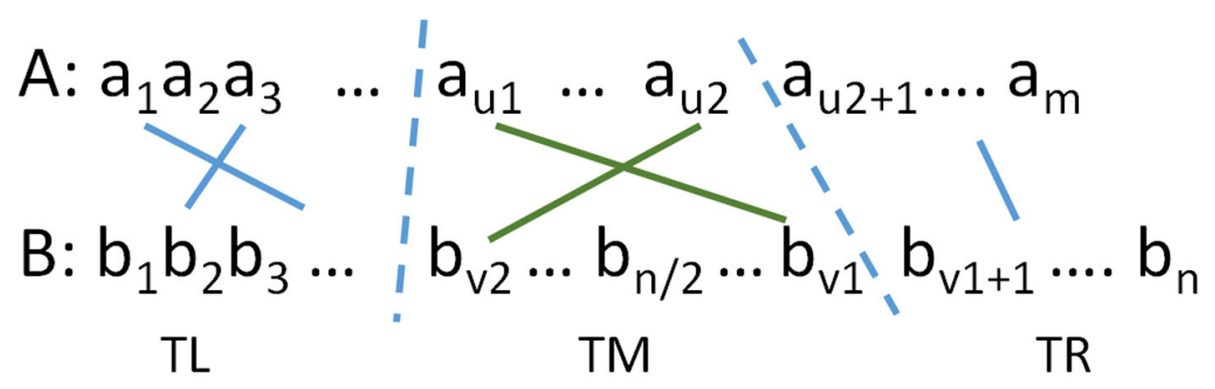

(b)

Fig. 3 Traces with and without center crossings [18]. a No center crossing. b With center crossing 
Table 1 Memory usage for DL distance algorithms on Xeon4

\begin{tabular}{ll}
\hline Algorithm & Memory \\
\hline Lowrance and Wagner & $30100.88 \mathrm{MB}$ \\
LS_DL & $41.93 \mathrm{MB}$ \\
LS_DL2 & $8.82 \mathrm{MB}$ \\
LS_Strip & $40.98 \mathrm{MB}$ \\
LS_Strip2 & $8.73 \mathrm{MB}$ \\
\hline
\end{tabular}

Jaro distance [16], only transpositions are permitted. The correct distance metric to use depends on the application. In the applications cited above, the DL distance is used as all 4 edit operations are permitted.

Lowrance and Wagner [17] considered a generalization of DL distance to the case when substitutions, inserts, deletes, and transpositions have different costs. Through a suitable choice of weights, the weighted DL distance can be made equal to the DL distance, Levenshtein distance, Hamming distance, and Jaro distance.

Lowrance and Wagner [17] have developed an $O(m n)$ time and $O(m n)$ space algorithm to find the minimum cost edit sequence (ie., sequence of substitutions, inserts, deletes, and transpositions) that transforms a given string of length $m$ into a given string of length $n$ provided that $2 T \geq I+D$, where $T, I$, and $D$, are respectively, the cost of a transposition, insertion, and deletion. In the DL distance, $T=I=D=1$ and so, $2 T=I+D$. Hence, the algorithm of Lowrance and Wagner [17] may be used to compute the DL distance as well as the corresponding edit sequence in $O(m n)$ time and $O(m n)$ space. This observation has also been made in [2]. In [18] we developed algorithms that run in $O(m n)$ time using only $O(s * \min \{m, n\}+m+n)$ space, where $s$ is the size of the alphabet comprising the strings, to compute the DL distance as well as the corresponding edit sequence. Since $s<<m$ and $s<<n$ in most applications (e.g., $s=20$ for protein sequences), this reduction in space enables the solution of much larger instances than is possible using the algorithm of [17]. Our algorithms in [18] are much faster as well. In this paper, we develop algorithms to compute the DL distance and corresponding edit sequence using $O(m+n)$ space and $O(m n)$ time. Extensive experimentation using 3 different platforms indicates that the algorithms of this paper are also faster than those of [18]. In fact, our fastest algorithm for the DL distance is up to $56.4 \%$ faster than the fastest algorithm in [18] when run on a single core. The single core speedup to find the corresponding edit sequence is up to $57.4 \%$. Our algorithms may be adapted to run on multicores providing a speedup of up to $59.3 \%$.

\section{DL dynamic programming recurrences}

Let $A[1: m]=a_{1} a_{2} \cdots a_{m}$ and $B[1: n]=b_{1} b_{2} \ldots b_{n}$ be two strings of length $m$ and $n$, respectively. Let $H_{i j}$ be the DL distance between $A[1: i]$ and $B[1: j]$. So, $H_{m n}$ is the DL distance between $A$ and $B$. The dynamic programming recurrence for $H$ is given below $[17,18]$.

$$
H_{i, 0}=i, H_{0, j}=j, 0 \leq i \leq m, 0 \leq j \leq n
$$

When $i>0$ and $j>0$,

$$
H_{i, j}=\min \left\{\begin{array}{l}
H_{i-1, j-1}+c\left(a_{i}, b_{j}\right) \\
H_{i, j-1}+1 \\
H_{i-1, j}+1 \\
H_{k-1, l-1}+(i-k-1)+1+(j-l-1)
\end{array}\right.
$$

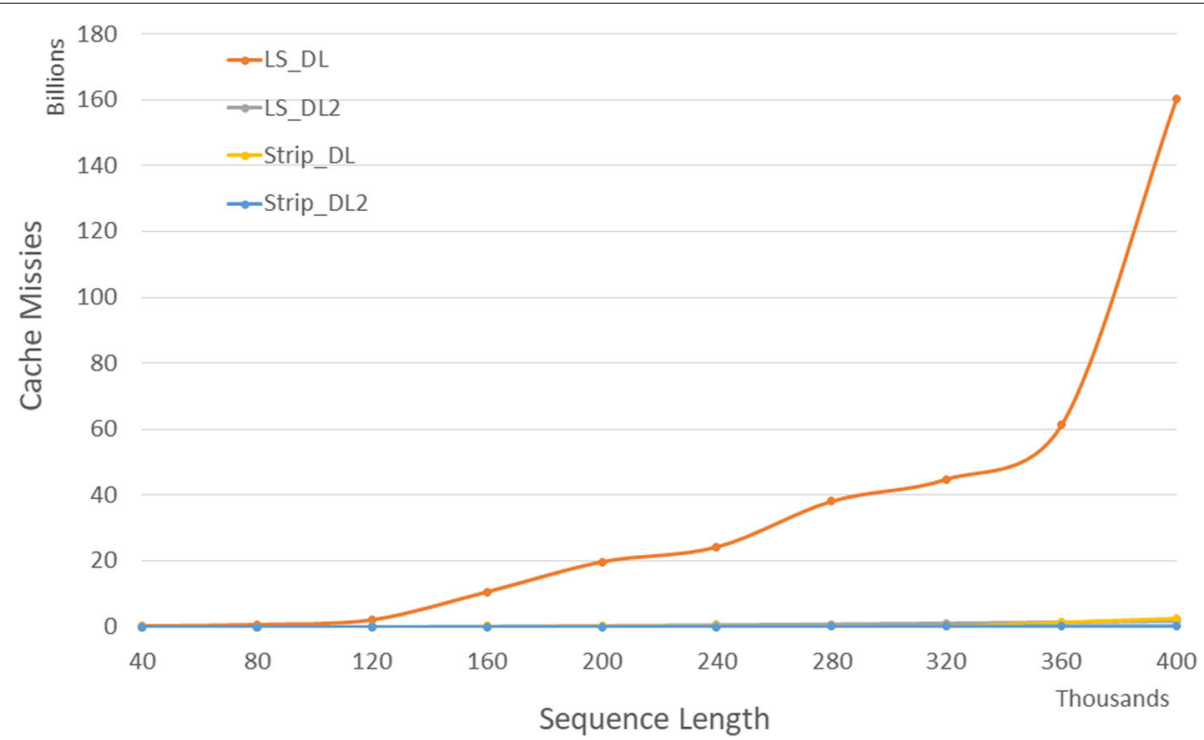

Fig. 4 Cache misses for DL distance algorithms, in billions, on Xeon4 
Table 2 Cache misses for DL distance algorithms, in millions, on Xeon4

\begin{tabular}{lllllllll}
\hline A & B & LS_DL & LS_DL2 & Strip_DL & Strip_DL2 & L vs L2 & S vs S2 & L2 vs S2 \\
\hline 40000 & 40000 & 265 & 14 & 6 & 1 & $94.9 \%$ & $76.6 \%$ & $90.4 \%$ \\
80000 & 80000 & 715 & 53 & 16 & 5 & $92.6 \%$ & $66.0 \%$ & $89.9 \%$ \\
120000 & 120000 & 2,180 & 121 & 42 & 11 & $94.4 \%$ & $73.1 \%$ & $90.7 \%$ \\
160000 & 160000 & 10,652 & 247 & 63 & 20 & $97.7 \%$ & $68.4 \%$ & $91.9 \%$ \\
200000 & 200000 & 19,751 & 397 & 147 & 32 & $98.0 \%$ & $78.0 \%$ & $91.9 \%$ \\
240000 & 240000 & 24,257 & 570 & 133 & 49 & $97.7 \%$ & $63.2 \%$ & $91.4 \%$ \\
280000 & 280000 & 38,119 & 781 & 188 & 66 & $98.0 \%$ & $65.0 \%$ & $91.6 \%$ \\
320000 & 320000 & 44,815 & 1,021 & 242 & 86 & $97.7 \%$ & $64.4 \%$ & $91.6 \%$ \\
360000 & 360000 & 61,296 & 1,290 & 1,352 & 111 & $97.9 \%$ & $91.8 \%$ & $91.4 \%$ \\
400000 & 400000 & 160,118 & 1,587 & 2,407 & 136 & $99.0 \%$ & $94.4 \%$ & $91.5 \%$ \\
\hline
\end{tabular}

where $c\left(a_{i}, b_{j}\right)$ is 1 if $a_{i} \neq b_{j}$ and 0 otherwise, $k=$ last $A[i]\left[b_{j}\right]$ is the last (i.e.,rightmost) occurrence of $b_{j}$ in $A$ that precedes position $i$ of $A$, and $l=\operatorname{last} B[j]\left[a_{i}\right]$ is the last occurrence of $a_{i}$ in $B$ that precedes position $j$ of $B$. When $k$ or $l$ do not exist, case 4 of Eq. 2 does not apply.

The four cases in Eq. 2 correspond to the four allowable edit operations: substitution, insertion, deletion and transposition. These cases are illustrated in Fig. 1, which depicts the possibilities for an optimal transformation of $A[1: i]$ to $B[1: j]$. Figure 1 a illustrates the first case, which is to optimally transform $A[1: i-1]$ into $B[1: j-1]$ and then substitute $b_{j}$ for $a_{i}$. If $a_{i}=b_{j}$, the substitution cost is 0 , otherwise it is 1 . Figure $1 \mathrm{~b}$ shows the second case. Here, $A[1: i]$ is optimally transformed into $B[1: j-1]$ and then $b_{j}$ is inserted at the end. In the third case (Fig. 1c)
$A[1: i-1]$ is optimally transformed into $B[1: j]$ and then $a_{i}$ is deleted. For the fourth and final case (Fig. 1d), assume that $k$ and $l$ exist. In this case, we are going to transpose $a_{k}$ and $a_{i}$. We first optimally transform $A[1: k-1]$ into $B[1: l-1]$. Since only adjacent characters may be transposed, the transposition of $a_{k}$ and $a_{i}$ must be preceded by a deletion of $a_{k+1}$ through $a_{i-1}$, which results in $a_{k}$ and $a_{i}$ becoming adjacent. Following the transposition, we insert $b_{l+1}$ through $b_{j-1}$ between the transposed $a_{k}$ and $a_{i}$, thereby transforming $A[1: i]$ into $B[1: j]$. The cost of optimally transforming $A[1: k-1]$ into $B[1: l-1]$ is $H_{k-1, l-1}$. The ensuing deletions have a cost of $i-k-1$ as this is the number of deletions performed, the transposition $a_{k}$ and $a_{i}$ costs 1 , and the final inserts cost $l-k-1$. So, the overall cost of case 4 is $H_{k-1, l-1}+(i-k-1)+1$ $+(j-l-1)$.

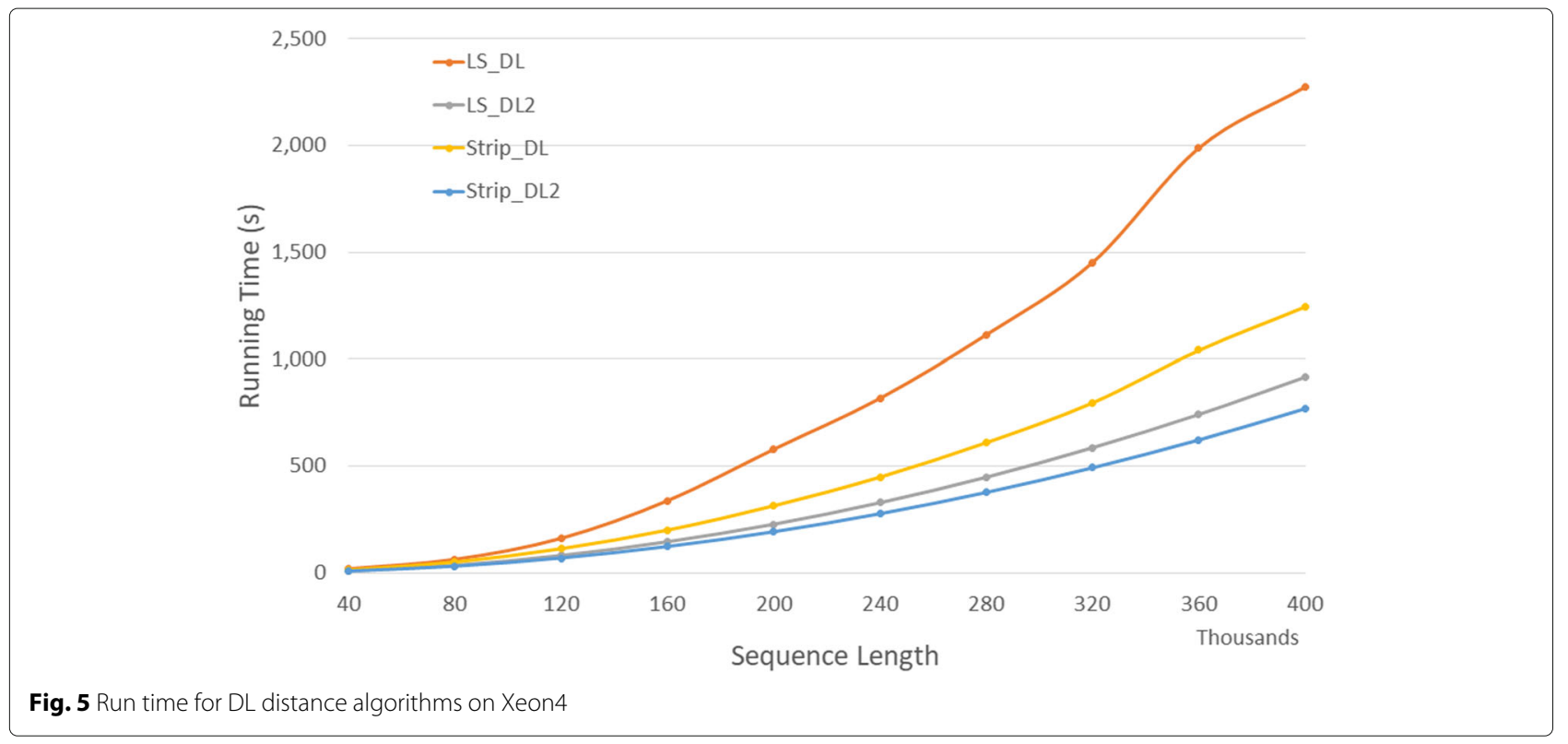


Table 3 Run time in $h h: m m$ : ss for DL distance algorithms on Xeon4

\begin{tabular}{lllllllll}
\hline A & B & LS_DL & LS_DL2 & Strip_DL & Strip_DL2 & L vs L2 & S vs S2 & L2 vs S2 \\
\hline 40000 & 40000 & $0: 00: 17$ & $0: 00: 09$ & $0: 00: 13$ & $0: 00: 08$ & $47.6 \%$ & $38.5 \%$ & $15.9 \%$ \\
80000 & 80000 & $0: 01: 02$ & $0: 00: 36$ & $0: 00: 50$ & $0: 00: 31$ & $41.3 \%$ & $38.1 \%$ & $15.4 \%$ \\
120000 & 120000 & $0: 02: 40$ & $0: 01: 22$ & $0: 01: 52$ & $0: 01: 09$ & $48.9 \%$ & $38.3 \%$ & $15.4 \%$ \\
160000 & 160000 & $0: 05: 37$ & $0: 02: 26$ & $0: 03: 19$ & $0: 02: 03$ & $56.6 \%$ & $38.2 \%$ & $15.7 \%$ \\
200000 & 200000 & $0: 09: 38$ & $0: 03: 48$ & $0: 05: 14$ & $0: 03: 12$ & $60.6 \%$ & $38.7 \%$ & $15.5 \%$ \\
240000 & 240000 & $0: 13: 37$ & $0: 05: 29$ & $0: 07: 28$ & $0: 04: 37$ & $59.8 \%$ & $38.2 \%$ & $15.7 \%$ \\
280000 & 280000 & $0: 18: 34$ & $0: 07: 28$ & $0: 10: 10$ & $0: 06: 17$ & $59.8 \%$ & $38.2 \%$ & $15.7 \%$ \\
320000 & 320000 & $0: 24: 13$ & $0: 09: 45$ & $0: 13: 17$ & $0: 08: 13$ & $59.7 \%$ & $38.2 \%$ & $15.8 \%$ \\
360000 & 360000 & $0: 33: 10$ & $0: 12: 21$ & $0: 17: 22$ & $0: 10: 24$ & $62.8 \%$ & $40.1 \%$ & $15.8 \%$ \\
400000 & 400000 & $0: 37: 55$ & $0: 15: 15$ & $0: 20: 46$ & $0: 12: 50$ & $59.8 \%$ & $38.2 \%$ & $15.8 \%$ \\
\hline
\end{tabular}

The algorithm of Lowrance and Wagner [17] computes $H_{m, n}$ using a $m \times n$ array for $H$ and one-dimensional arrays of size $s$ for last $A$ and last $B$, where $s$ is the size of the alphabet from which the strings $A$ and $B$ are drawn. It computes the $H_{i, j}$ 's by rows beginning with row 1 and within a row, the elements are computed left-to-right by columns. While algorithm $L S \_D L$ of [18] also computes $H$ by rows and within a row by columns left-to-right, it does this using a one-dimensional array of size $n$ for the current row being computed, a one-dimensional array of size $s$ for last $A$, and an $s \times n$ array $T$ with the property that if $w$ is the last row of $H$ computed so far such that $A[w]=c$, then $T[c][*]=H[w-1][*]$. As noted in [18] when $m<n$, we may swap the strings $A$ and $B$ resulting in a space requirement of $O(s \min \{m, n\}+n)$. The time complexity of $L S \_D L$ is $O(m n)$. A cache-efficient version, Strip_DL, of $L S \_D L$ that computes $H$ by strips whose width is no larger than the cache size is also developed in [18]. This cache efficient algorithm has the same asymptotic time and space complexities as does $L S \_D L$. But, as demonstrated in [18], Strip_DL is much faster than $L S \_D L$.

The linear space algorithms we develop in this paper use a refined dynamic programming recurrence for $H$. We make the observation that when either $a_{i}=b_{j}$ or $\min \{i-$ $k, j-l\} \geq 2$ in the fourth case of Eq. 2 , then it is sufficient to consider only the first three cases. To see this, note that when $a_{i}=b_{j}$ the transposition of $a_{k}$ and $a_{i}$ done following the deletion of $a_{k+1}$ through $a_{i-1}$ in case 4 (Fig. 1d) is unnecessary as $a_{k}=b_{j}=a_{i}$. So, one of the first three cases has to result in a smaller value than case 4 . Next, consider the case when $a_{i} \neq b_{j}$ and $\min \{i-k, j-l\} \geq 2$. Suppose that $2 \leq i-k \leq j-l$. The cost of transforming $A[1: i]$ to $B[1: j]$ by using an optimal transformation of $A[1: k-1]$

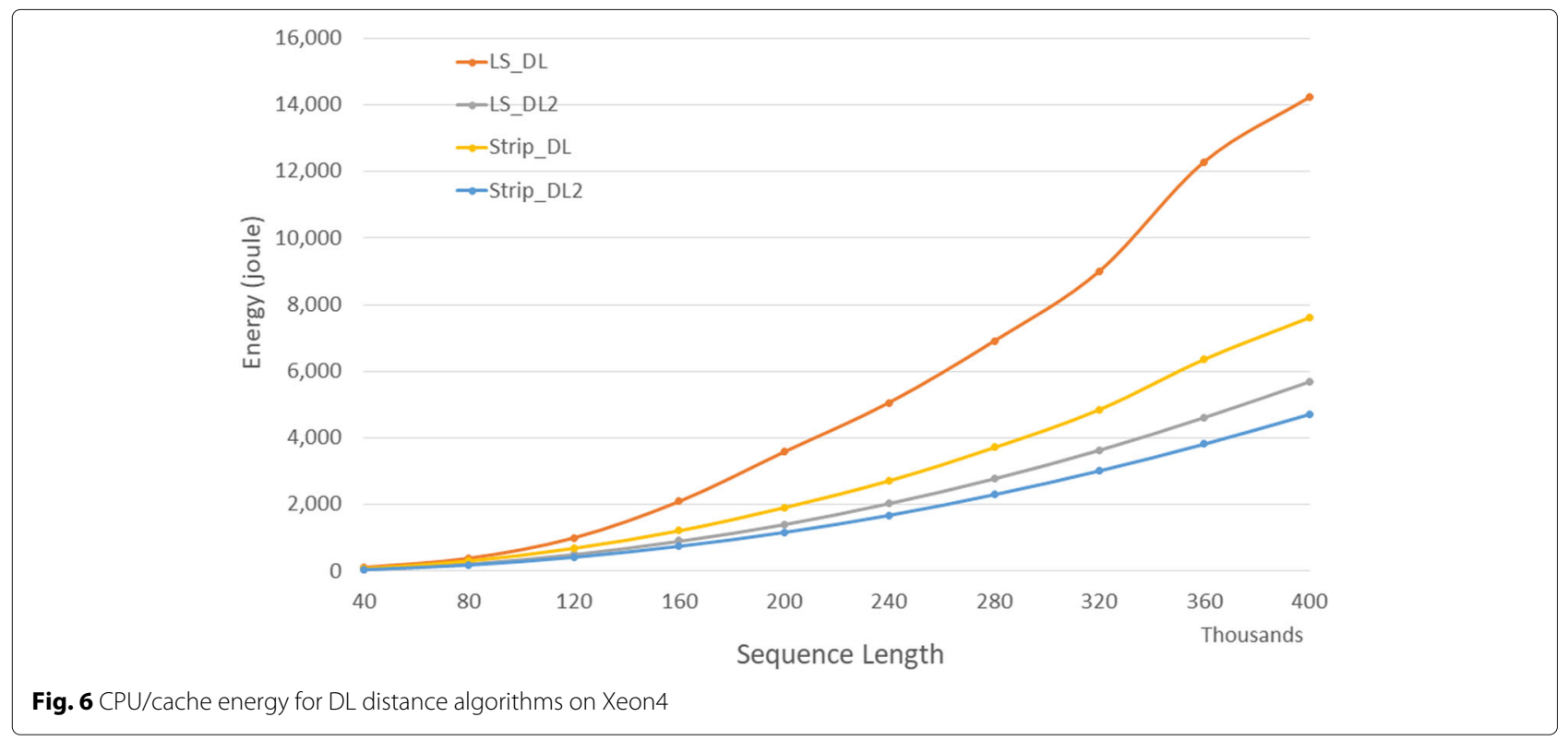


Table 4 CPU/cache energy in joules for DL distance algorithms on Xeon4

\begin{tabular}{lllllllll}
\hline A & B & LS_DL & LS_DL2 & Strip_DL & Strip_DL2 & Lvs L2 & S vs S2 & L2 vs S2 \\
\hline 40000 & 40000 & 107 & 56 & 77 & 47 & $47.3 \%$ & $39.0 \%$ & $17.1 \%$ \\
80000 & 80000 & 384 & 225 & 305 & 187 & $41.5 \%$ & $38.7 \%$ & $16.7 \%$ \\
120000 & 120000 & 997 & 509 & 687 & 422 & $49.0 \%$ & $38.5 \%$ & $17.0 \%$ \\
160000 & 160000 & 2088 & 907 & 1212 & 752 & $56.6 \%$ & $38.0 \%$ & $17.1 \%$ \\
200000 & 200000 & 3577 & 1406 & 1906 & 1167 & $60.7 \%$ & $38.8 \%$ & $17.0 \%$ \\
240000 & 240000 & 5058 & 2040 & 2714 & 1680 & $59.7 \%$ & $38.1 \%$ & $17.6 \%$ \\
280000 & 280000 & 6906 & 2781 & 3711 & 2302 & $59.7 \%$ & $38.0 \%$ & $17.2 \%$ \\
320000 & 320000 & 9000 & 3638 & 4852 & 3016 & $59.6 \%$ & $37.9 \%$ & $17.1 \%$ \\
360000 & 360000 & 12287 & 4619 & 6366 & 3822 & $62.4 \%$ & $40.0 \%$ & $17.3 \%$ \\
400000 & 400000 & 14218 & 5690 & 7615 & 4712 & $60.0 \%$ & $38.1 \%$ & $17.2 \%$ \\
\hline
\end{tabular}

to $B[1: l-1]$ and then doing $j-l+1$ substitutions and inserts is $H_{k-1, l-1}+j-l+1$. Doing the transformation as is case 4 has a cost $H_{k-1, l-1}+(i-k-1)+1+(j-l-1)$ $\geq H_{k-1, l-1}+j-l+1$. So, doing the transposition (case 4 ) isn't any better than using only substitutions and inserts. Hence, case 4 need not be considered. The case when $2 \leq j-l \leq i-k$ is symmetric.

The preceding observation establishes the correctness of the following refined recurrence for $H$.

$$
H_{i, 0}=i, H_{0, j}=j, 0 \leq i \leq m, 0 \leq j \leq n
$$

When $i>0$ and $j>0$,

$$
H_{i, j}=\min \left\{\begin{array}{l}
H_{i-1, j-1}+c\left(a_{i}, b_{j}\right) \\
H_{i, j-1}+1 \\
H_{i-1, j}+1 \\
\left\{\begin{array}{c}
H_{k-1, j-2}+(i-k) \\
\text { if } j-l=1 \text { and } a_{i} \neq b_{j} \\
H_{i-2, l-1}+(j-l) \\
\text { if } i-k=1 \text { and } a_{i} \neq b_{j} \\
\infty \text { otherwise }
\end{array}\right.
\end{array}\right.
$$

where $c\left(a_{i}, b_{j}\right)$ is 1 if $a_{i} \neq b_{j}$ and 0 otherwise, $k=$ last $A[i]\left[b_{j}\right]$ and $l=$ last $B[j]\left[a_{i}\right]$.

We observe that the above refined recurrence for $H$ holds even in the weighted setting provided that $2 S \leq$ $I+D \leq 2 T$, where $S, I, D$, and $T$ are, respectively, the cost of a substitution, insertion, deletion, and transposition; the cost of a substitution is $>0$ when the characters involved are different and 0 when these are the same. This observation follows from the following.

When $a_{i}=b_{j}, S=0$

$$
\begin{aligned}
& H_{k-1, l-1}+(i-k-1) D+T+(j-l-1) I \\
& =H_{k, l}+(i-k-1) D+T+(j-l-1) I \\
& \geq H_{i-1, l}+T+(j-l-1) I \\
& \geq H_{i-1, j-1} \\
& =H_{i, j}
\end{aligned}
$$

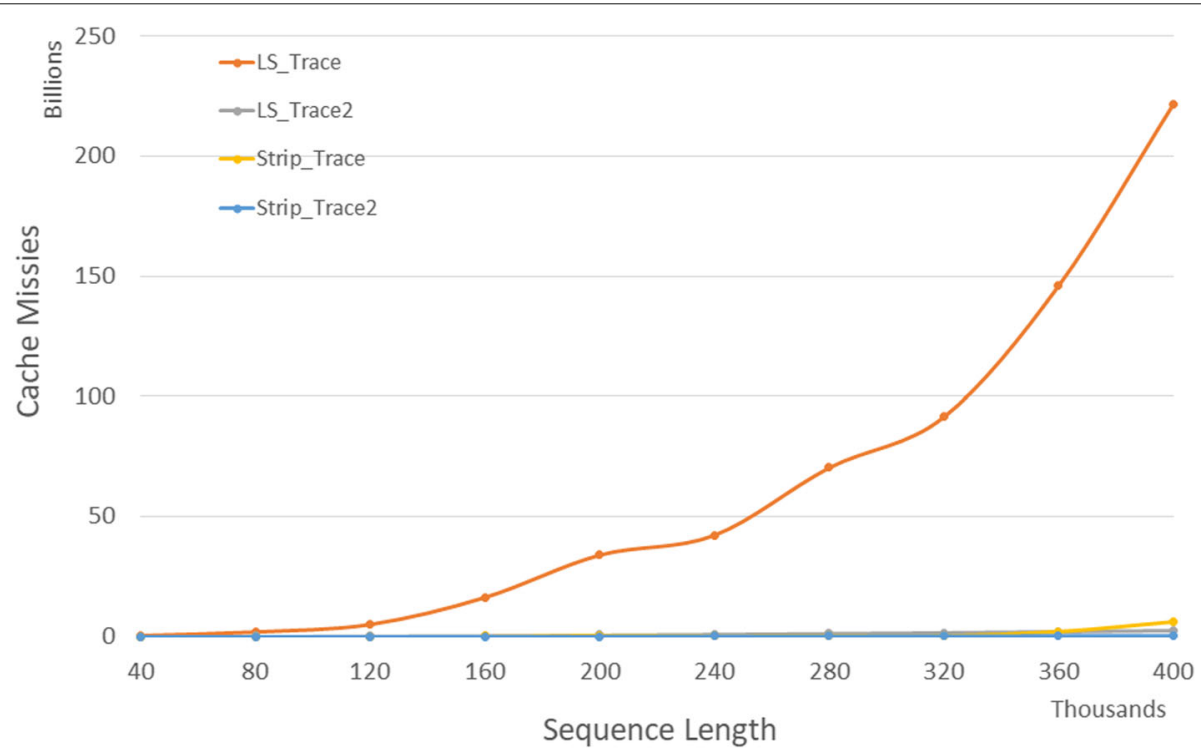

Fig. 7 Cache misses for DL trace algorithms on Xeon4 
Table 5 Cache misses in millions for DL trace algorithms on Xeon4

\begin{tabular}{lllllllll}
\hline A & B & LS_Trace & LS_Trace2 & Strip_Trace & Strip_Trace2 & L vs L2 & S vs S2 & L2 vs S2 \\
\hline 40000 & 40000 & 423 & 20 & 24 & 3 & $95.2 \%$ & $88.1 \%$ & $86.2 \%$ \\
80000 & 80000 & 1,970 & 89 & 29 & 12 & $95.5 \%$ & $58.5 \%$ & $86.6 \%$ \\
120000 & 120000 & 5,100 & 212 & 66 & 25 & $95.8 \%$ & $61.8 \%$ & $88.1 \%$ \\
160000 & 160000 & 16,350 & 403 & 115 & 44 & $97.5 \%$ & $61.9 \%$ & $89.1 \%$ \\
200000 & 200000 & 33,998 & 622 & 513 & 72 & $98.2 \%$ & $85.9 \%$ & $88.3 \%$ \\
240000 & 240000 & 42,252 & 897 & 268 & 101 & $97.9 \%$ & $62.4 \%$ & $88.8 \%$ \\
280000 & 280000 & 70,370 & 1,244 & 358 & 139 & $98.2 \%$ & $61.3 \%$ & $88.9 \%$ \\
320000 & 320000 & 91,501 & 1,576 & 453 & 181 & $98.3 \%$ & $60.1 \%$ & $88.5 \%$ \\
360000 & 360000 & 146,103 & 2,001 & 2,120 & 230 & $98.6 \%$ & $89.1 \%$ & $88.5 \%$ \\
400000 & 400000 & 221,690 & 2,435 & 6,032 & 276 & $98.9 \%$ & $95.4 \%$ & $88.6 \%$ \\
\hline
\end{tabular}

When $2 \leq i-k \leq j-l$

$$
\begin{aligned}
& H_{k-1, l-1}+(i-k-1) D+T+(j-l-1) I \\
& \geq H_{k-1, l-1}+(i-k-1)(2 S-I)+S+(j-l-1) I \\
& =H_{k-1, l-1}+(i-k+1) S+((j-l)-(i-k)) I
\end{aligned}
$$

The case when $2 \leq j-l \leq i-k$ is symmetric.

The algorithms developed in this paper are based on our refined recurrence for $H$.

\section{Methods}

\section{DL distance algorithms}

In this section, we develop two algorithms, $L S \_D L 2$ and Strip_DL2, to compute the DL distance between two strings of length $m$ and $n$ drawn from an alphabet of size $s$. We note that when $s>m+n$, at least $s-m-n$ characters of the alphabet appear in neither $A$ nor $B$. So, these nonappearing characters may be removed from the alphabet and we can work with this reduced size alphabet. Hence, throughout this paper, we assume that $s \leq m+n$. Our algorithms, which take $O(m+n)$ space, are based on the recurrence of Eqs. 3 and 4 and are the counterparts of algorithms $L S \_D L$ and Strip_DL of [18] that are based on the recurrence of Eqs. 1 and 2.

\section{Algorithm LS_DL2}

Like algorithm $L S \_D L$ of [18], $L S \_D L 2$ (Algorithm 1) computes $H$ by rows from top to bottom and within a row

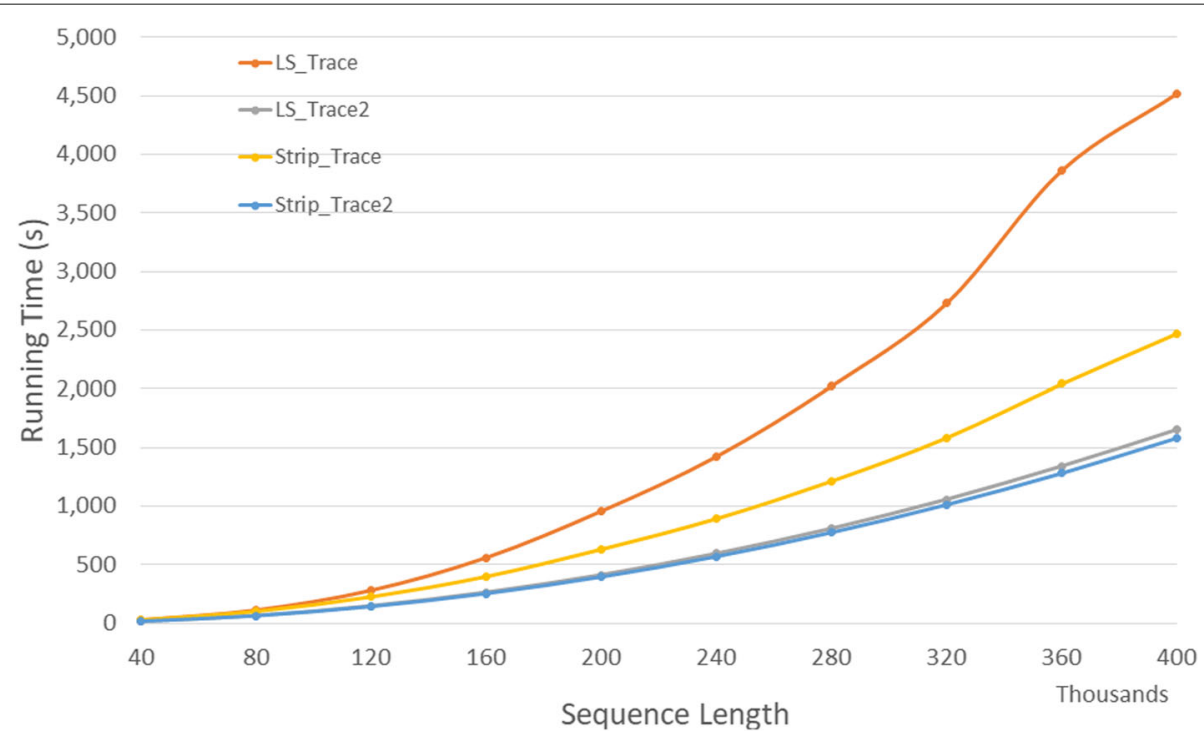

Fig. 8 Run time for DL trace algorithms on Xeon4 
Table 6 Run time in $h h$ : $m m$ : ss for DL trace algorithms on Xeon4

\begin{tabular}{lllllllll}
\hline A & B & LS_Trace & LS_Trace2 & Strip_Trace & Strip_Trace2 & L vs L2 & S vs S2 & L2 vs S2 \\
\hline 40000 & 40000 & $0: 00: 30$ & $0: 00: 17$ & $0: 00: 26$ & $0: 00: 16$ & $44.0 \%$ & $37.8 \%$ & $3.6 \%$ \\
80000 & 80000 & $0: 01: 54$ & $0: 01: 06$ & $0: 01: 40$ & $0: 01: 04$ & $42.1 \%$ & $36.5 \%$ & $3.8 \%$ \\
120000 & 120000 & $0: 04: 42$ & $0: 02: 29$ & $0: 03: 44$ & $0: 02: 23$ & $47.3 \%$ & $36.2 \%$ & $4.0 \%$ \\
160000 & 160000 & $0: 09: 21$ & $0: 04: 25$ & $0: 06: 37$ & $0: 04: 14$ & $52.8 \%$ & $36.1 \%$ & $4.2 \%$ \\
200000 & 200000 & $0: 15: 58$ & $0: 06: 53$ & $0: 10: 30$ & $0: 06: 36$ & $56.8 \%$ & $37.1 \%$ & $4.1 \%$ \\
240000 & 240000 & $0: 23: 42$ & $0: 09: 56$ & $0: 14: 52$ & $0: 09: 31$ & $58.1 \%$ & $36.0 \%$ & $4.2 \%$ \\
280000 & 280000 & $0: 33: 41$ & $0: 13: 29$ & $0: 20: 13$ & $0: 12: 57$ & $60.0 \%$ & $36.0 \%$ & $4.1 \%$ \\
320000 & 320000 & $0: 45: 26$ & $0: 17: 37$ & $0: 26: 24$ & $0: 16: 54$ & $61.2 \%$ & $36.0 \%$ & $4.1 \%$ \\
360000 & 360000 & $1: 04: 18$ & $0: 22: 21$ & $0: 34: 01$ & $0: 21: 23$ & $65.2 \%$ & $37.1 \%$ & $4.3 \%$ \\
400000 & 400000 & $1: 15: 14$ & $0: 27: 33$ & $0: 41: 11$ & $0: 26: 24$ & $63.4 \%$ & $35.9 \%$
\end{tabular}

by columns from left to right. For convenience, we augment $H$ with row -1 and column -1 . All values on this row and on this column are maxVal, where maxVal is a large value. Algorithm $L S \_D L 2$ uses 4 one-dimensional arrays last_row_id $[1: s], R[-1: n], R 1[-1: n]$, and $F R[-1: n]$ and a few simple variables that have the following semantics when we are computing $H_{i j}$. $k$ and $l$ are as in Eqs. 4.

1. $F R[q]=H_{k-1, q-2}$ for the current $i$ in case $q \geq j$ and for the next $i$ in case $q<j$

2. $R[q]=H_{i, q}$ if $q<j$ and $H_{i-2, q}$ if $q \geq j$.

3. $R 1[q]=H_{i-1, q}$

4. last_row_id $[c]=$ largest $k<i$ such that $A[k]=c$

5. last_col_id $=$ largest $l<j$ such that $B[l]=A[i]$

6. $T$ is the value to use for $H_{i-2, l-1}$ should this be needed in the computation of $H_{i j}$
7. last_i2l1 $=H_{i-2, j-1}$

8. $\operatorname{diag}$... Case 1 of Eq. 4

9. left ... Case 2 of Eq. 4

10. up ... Case 3 of Eq. 4

11. transpose $\cdots$ Case 4 of Eq. 4

Lines 2 and 3 of the algorithm initialize $F R, R 1$, and $R$ so that following the swap of line $6, R 1[q]=H_{0, q}$ and $R[q]=$ $H_{-1, q},-1 \leq q \leq n$. In other words, at the start of iteration $i=1$ of the for loop of lines 9-30, $R 1$ and $R$, respectively, correspond to rows $i-1$ (i.e., row 0 ) and $i-2$ (i.e., row -1) of $H$. At this time, $F R[-1: n]=$ maxValue, which corresponds to the initial situation that $k=\operatorname{last} A[i]\left[b_{j}\right]$ is undefined. This will be updated as $l a s t A[i]\left[b_{j}\right]$ gets defined. last_row_id $[1: s]$ is initialized to -1 in line 4 for each character $c$ to indicate the fact that at the start of the $i=1$ loop, $A[p], p<1$ isn't a character of the

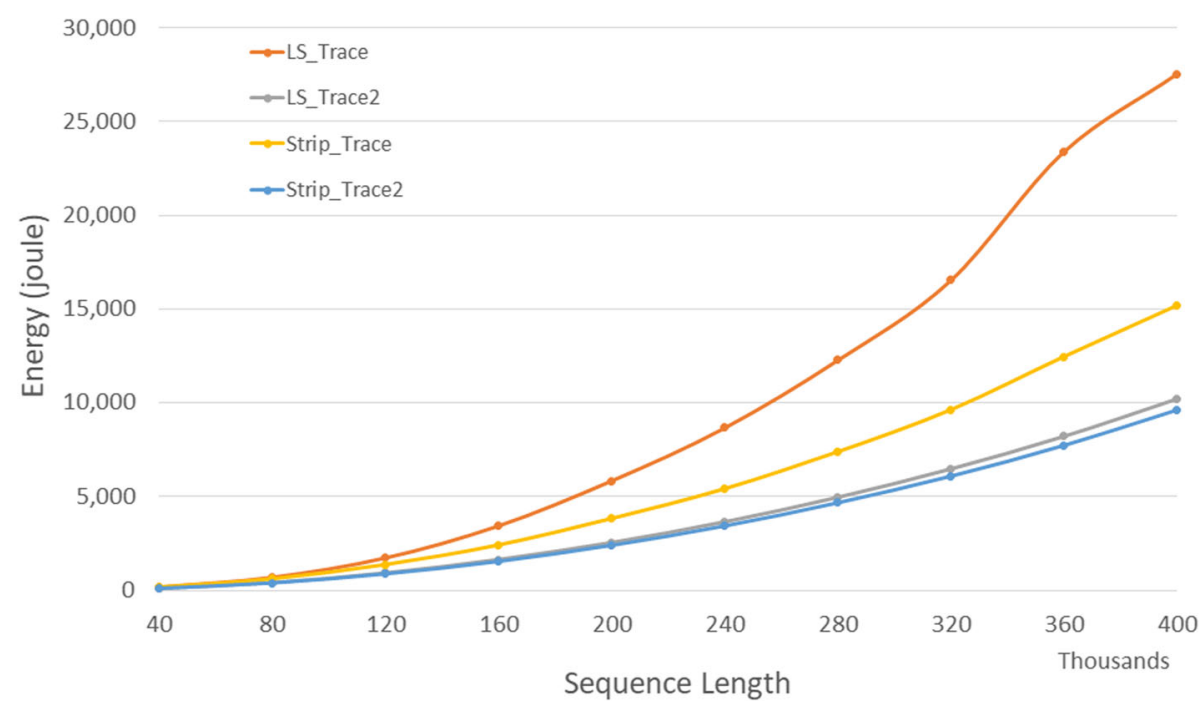

Fig. 9 CPU/cache energy for DL trace algorithms on Xeon4 
Table 7 CPU/cache energy in joules for DL trace algorithms on Xeon4

\begin{tabular}{lllllllll}
\hline A & B & LS_Trace & LS_Trace2 & Strip_Trace & Strip_Trace2 & Lvs L2 & S vs S2 & L2 vs S2 \\
\hline 40000 & 40000 & 181.4 & 103.1 & 157.0 & 97.8 & $43.2 \%$ & $37.7 \%$ & $5.2 \%$ \\
80000 & 80000 & 703.1 & 413.8 & 610.7 & 389.3 & $41.1 \%$ & $36.3 \%$ & $5.9 \%$ \\
120000 & 120000 & $1,736.9$ & 929.9 & $1,365.3$ & 873.4 & $46.5 \%$ & $36.0 \%$ & $6.1 \%$ \\
160000 & 160000 & $3,443.8$ & $1,644.4$ & $2,407.9$ & $1,540.3$ & $52.3 \%$ & $36.0 \%$ & $6.3 \%$ \\
200000 & 200000 & $5,843.6$ & $2,556.4$ & $3,818.7$ & $2,398.1$ & $56.3 \%$ & $37.2 \%$ & $6.2 \%$ \\
240000 & 240000 & $8,665.3$ & $3,659.6$ & $5,403.6$ & $3,430.8$ & $57.8 \%$ & $36.5 \%$ & $6.3 \%$ \\
280000 & 280000 & $12,275.0$ & $4,970.5$ & $7,372.3$ & $4,665.3$ & $59.5 \%$ & $36.7 \%$ & $6.1 \%$ \\
320000 & 320000 & $16,536.9$ & $6,486.5$ & $9,609.6$ & $6,084.6$ & $60.8 \%$ & $36.7 \%$ & $6.2 \%$ \\
360000 & 360000 & $23,396.4$ & $8,224.1$ & $12,439.7$ & $7,725.9$ & $64.8 \%$ & $37.9 \%$ & $6.1 \%$ \\
400000 & 400000 & $27,551.9$ & $10,229.2$ & $15,167.8$ & $9,616.6$ & $62.9 \%$ & $36.6 \%$ & $6.0 \%$ \\
\hline
\end{tabular}

alphabet. last_col_id is set to -1 at the start of each iteration of the loop of lines 5-32 as at the start of this loop, no character of $B$ has been examined and so there is no last seen occurrence of $A[i]$ in $B$ (for this iteration of the for loop of lines 5-32). Also, at the start of the computation of each row of $H$, last_i $2 l 1$ is set to $R[0]$, because, by the semantics of last_i2l1, when we are computing $H_{i, 1}$, last_i2l1 $=H_{i-2,0}=R[0]$. Following this, $R[0]$ is set to $i$ to indicate that the cheapest way to transform $A[1: i]$ into $B[0: 0]$ is to do $i$ deletes at a total cost of $i$ (hence, when we are computing $H_{i, 1}$ in the loop of lines 9-30, $R[q]=H_{i j}$, $q<1$ ), and $T$ is set to maxVal as when we start a row computation, $l$ is undefined. So, the initializations establish the variable semantics given above.

In lines 10-12 of the loop of lines 9-30, diag, left, and up are set to the values specified in cases 1-3 of Eq. 4 . Note that from the semantics of the variables, $R 1[j-1]=$ $H_{i-1, j-1}, R[j-1]=H_{i, j-1}$, and $R 1[j]=H_{i-1, j}$ at this time. Line 13 computes the minimum of the terms in the first 3 cases of Eq. 4. If $A[i]=B[j]$ (line 14), then $H_{i j}$ is determined by cases $1-3$ and the value of temp computed in line 13 is $H_{i, j}$. At this time, we need to update last_col_id as the most recently seen occurrence of $A[i]$ is now at position $j$ of $B$. Since $A[i]=B[j]$, last $A[i+1]\left[b_{j}\right]=i$. So, the $H_{k-1, j-2}$ to use for the next $i$ in case 4 is $H_{i-1, j-2}=$ $R 1[j-2]$. This value is saved in $F R[j]$ in line 16 . Since $A[i]=B[j]$, the value to use for $H_{i-2, l-1}$ in case 4 of Eq. 4 in future iterations of the for $j$ loop (until, of course, we encounter another $j$ where $A[i]=B[j])$ becomes $H_{i-2, j-1}$, which by the variable semantics is last_i2l1. This value is saved in $T$ in line 17 .

When $A[i] \neq B[j]$, lines 19-26 are executed. From the semantics of last_row_id and last_col_id, it follows that line 19 correctly sets $k$ and $l$. Lines 22 and 24, respectively, compute the cost of case 4 when $j-l=1$ and $i-k=$ 1 , respectively. Note that by the semantics of $F R$ and $T$, $F R[j]=H_{k-1, j-2}$ and $T=H_{i-2, l-1}$. So, lines 22 and 25

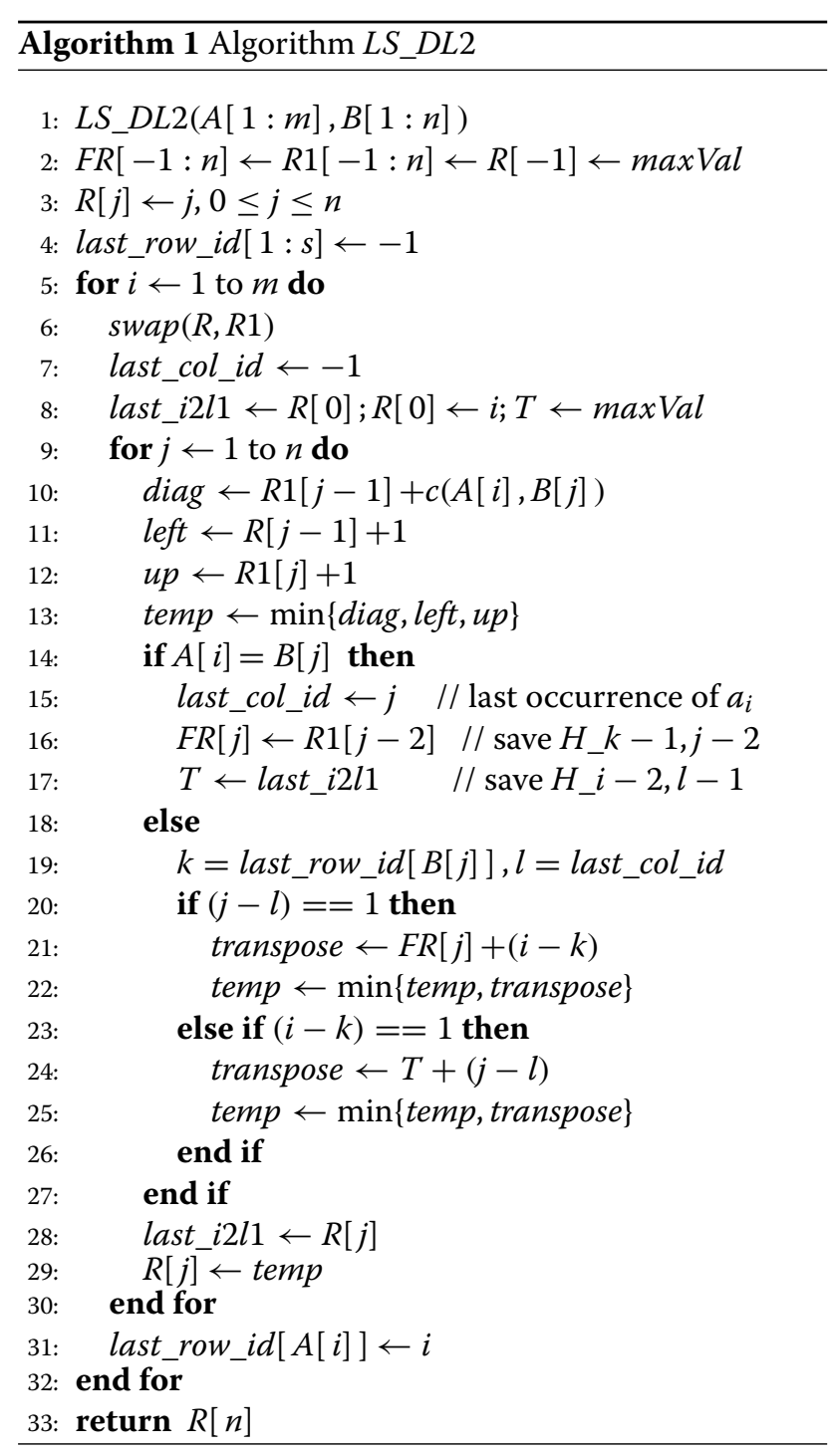




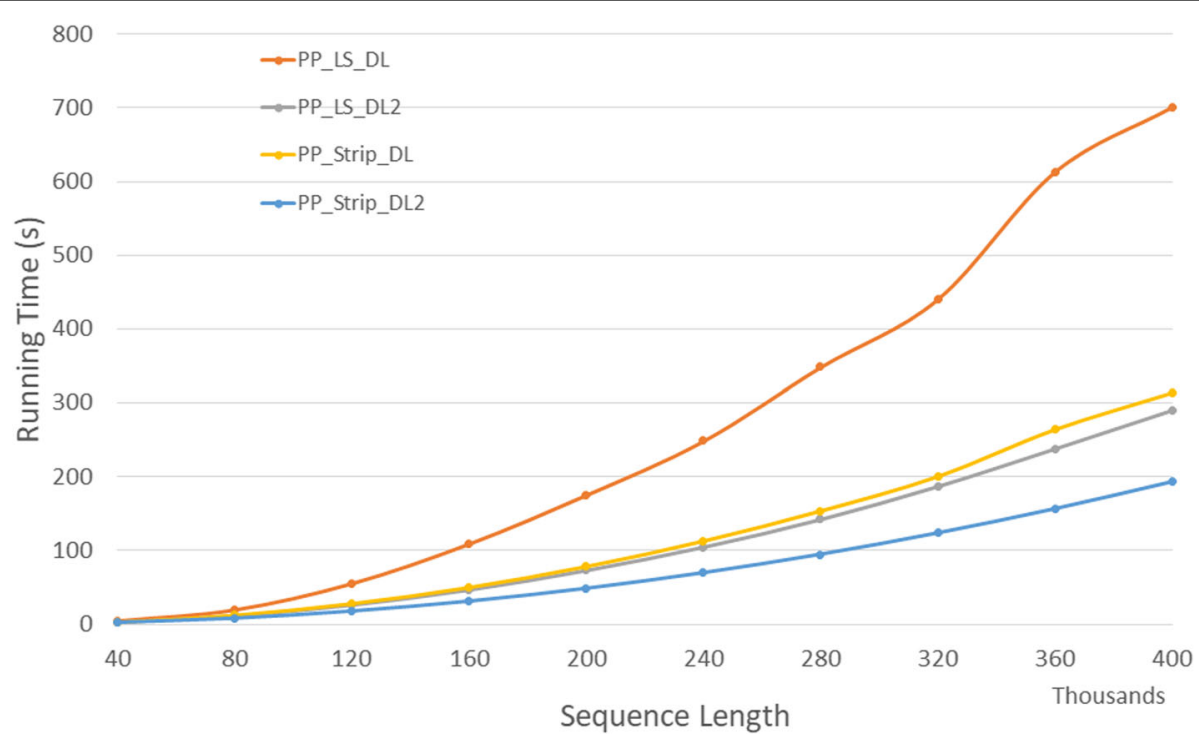

Fig. 10 Run time of parallel DL distance algorithms, in seconds, on Xeon4

update temp to be $H_{i, j}$. When we reach line 29, regardless of whether $A[i]=B[j]$ or $A[i] \neq B[j], R[j]=H_{i-2, j}$ and temp $=H_{i, j}$.

In line 28 , we set last_i2l1 $=R[j]=H_{i-2, j}$. While this momentarily upsets the semantics of last_i2l1, the semantics are restored upon the start of next iteration of the for $j$ loop as $j$ increases by 1 or in line 8 if the for $j$ loop terminates and we advance to the next iteration of the for $i$ loop. Line 29 sets $R[j]=H_{i j}$, which similarly upsets the semantics of $R$ but correctly sets up the semantics for the next iteration. Finally, line 31 correctly updates last_row_id so as to preserve its semantics for the next iteration of the for $i$ loop.

The correctness of $L S \_D L 2$ follows from the preceding discussion. The space and time complexities are readily seen to be $O(m+n+s)=O(m+n)$ and
$O(m n+s)=O(m n)$, respectively. When $m<n$, the space complexity may be reduced by a constant factor by swapping $A$ and $B$. Using the LRU cache model of [18], one may show that $L S \_D L 2$ has approximately $3 m n / w$ cache misses, where $w$ is the width of a cache line. By comparison, the number of cache misses for $L S \_D L$ is $m n(1+3 / w)$.

\section{Strip algorithm Strip_DL2}

As in [18], we can reduce cache misses, which in turn reduces run time, by partitioning $H$ into $n / q$ strips of size $m \times q$, where $q$ is the largest strip width for which the data needed in the computation of the strip fits into the cache. $H$ is computed by strips from left to right and the computation of each strip is done using $L S \_D L 2$. To enable this computation by strips, one strip needs to pass computed

Table 8 Run time of parallel DL distance algorithms, in $h h: m m: s S$, on Xeon4

\begin{tabular}{lllllllll}
\hline A & B & PP_LS_DL & PP_LS_DL2 & PP_Strip_DL & PP_Strip_DL2 & L vs L2 & S vs S2 & L2 vs S2 \\
\hline 40000 & 40000 & $0: 00: 05$ & $0: 00: 03$ & $0: 00: 03$ & $0: 00: 02$ & $42.4 \%$ & $38.1 \%$ & $33.8 \%$ \\
80000 & 80000 & $0: 00: 20$ & $0: 00: 12$ & $0: 00: 13$ & $0: 00: 08$ & $41.8 \%$ & $38.1 \%$ & $33.6 \%$ \\
120000 & 120000 & $0: 00: 56$ & $0: 00: 26$ & $0: 00: 28$ & $0: 00: 17$ & $52.8 \%$ & $38.5 \%$ & $33.8 \%$ \\
160000 & 160000 & $0: 01: 49$ & $0: 00: 47$ & $0: 00: 50$ & $0: 00: 31$ & $57.2 \%$ & $38.3 \%$ & $33.9 \%$ \\
200000 & 200000 & $0: 02: 55$ & $0: 01: 13$ & $0: 01: 19$ & $0: 00: 48$ & $58.3 \%$ & $38.4 \%$ & $33.9 \%$ \\
240000 & 240000 & $0: 04: 09$ & $0: 01: 45$ & $0: 01: 53$ & $0: 01: 10$ & $57.9 \%$ & $38.3 \%$ & $33.5 \%$ \\
280000 & 280000 & $0: 05: 48$ & $0: 02: 22$ & $0: 02: 34$ & $0: 01: 35$ & $59.1 \%$ & $38.3 \%$ & $33.4 \%$ \\
320000 & 320000 & $0: 07: 21$ & $0: 03: 07$ & $0: 03: 20$ & $0: 02: 04$ & $57.5 \%$ & $38.2 \%$ & $33.8 \%$ \\
360000 & 360000 & $0: 10: 13$ & $0: 03: 58$ & $0: 04: 24$ & $0: 02: 37$ & $61.2 \%$ & $40.6 \%$ & $34.2 \%$ \\
400000 & 400000 & $0: 11: 41$ & $0: 04: 50$ & $0: 05: 13$ & $0: 03: 14$ & $58.6 \%$ & $38.2 \%$ & $33.3 \%$ \\
\hline
\end{tabular}




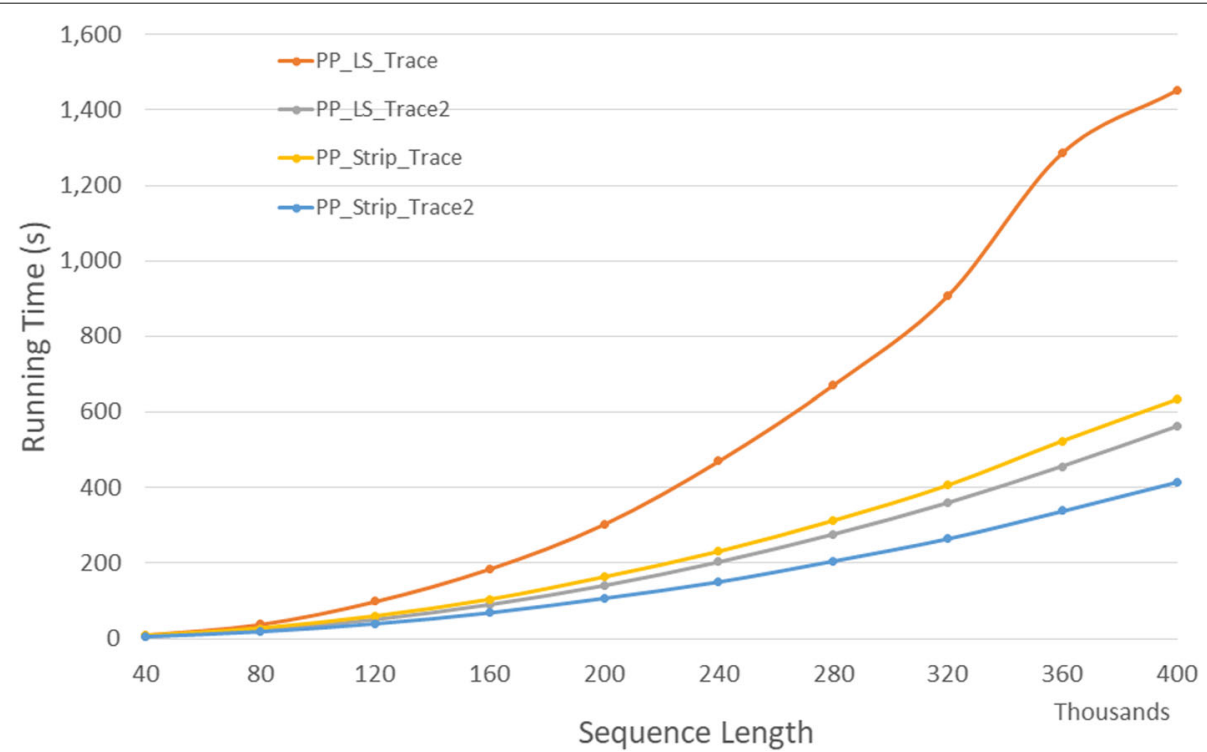

Fig. 11 Run time of parallel DL trace algorithms, in seconds, on Xeon4

values to the next using three additional one-dimensional arrays $C, C 1$, and $F C$ of size $m$ each. $C$ records the values of $H$ computed for the rightmost column in the strip; $C 1$ records the values of $H$ computed for the next to rightmost column in the strip; and $F C[i]$ is the value of $T$ (i.e., $H[i-2][l-1]$ ) at row $i$, where $l$ is the last column where $B[l]=A[i]$ in the strip. We name this new algorithm as Strip_DL2. The space complexity of Strip_DL2 is $O(m+n)$ and its time complexity is $O(m n)$. For the LRU cache model of [18] the number of cache misses is approximately $\frac{6 m n}{w q}$.

\section{DL trace algorithms}

Wagner and Fischer [19] introduced the concept of a trace to describe an edit sequence when the edit operations are limited to insert, delete, and substitute. Lowrance and Wagner [17] extended the concept of a trace to include transpositions. We reproduce here the definition and example used by us in [18]. A trace for the strings $A=a_{1} \cdots a_{m}$ and $B=b_{1} \cdots b_{n}$ is a set $T$ of lines, where the endpoints $u$ and $v$ of a line $(u, v)$ denote positions in $A$ and $B$, respectively. A set of lines $T$ is a trace iff:

1. For every $(u, v) \in T, u \leq m$ and $v \leq n$.

2. The lines in $T$ have distinct $A$ positions and distinct $B$ positions. That is, no two lines in $T$ have the same $u$ or the same $v$.

A line $(u, v)$ is balanced iff $a_{u}=b_{v}$ and two lines $\left(u_{1}, v_{1}\right)$ and $\left(u_{2}, v_{2}\right)$ cross iff $\left(u_{1}<u_{2}\right)$ and $\left(v_{1}>v_{2}\right)$. It is easy to see that $T=\{(1,2),(3,1),(4,3),(5,6)\}$ (see Fig. 2) is a trace for the strings $A=$ dafac and $B=$ fdbbec. Line $(4,3)$ is not balanced as $a_{4} \neq b_{3}$. The remaining 3 lines in the trace are balanced. The lines $(1,2)$ and $(3,1)$ cross.

Table 9 Run time of parallel DL trace algorithms, in $h h: m m: s s$, on Xeon4

\begin{tabular}{lllllllll}
\hline A & B & PP_LS_Trace & PP_LS_Trace2 & PP_Strip_Trace & PP_Strip_Trace2 & Lvs L2 & S vs S2 & L2 vs S2 \\
\hline 40000 & 40000 & $0: 00: 10$ & $0: 00: 06$ & $0: 00: 07$ & $0: 00: 05$ & $40.8 \%$ & $27.1 \%$ & $10.4 \%$ \\
80000 & 80000 & $0: 00: 38$ & $0: 00: 23$ & $0: 00: 27$ & $0: 00: 19$ & $39.9 \%$ & $30.7 \%$ & $19.2 \%$ \\
120000 & 120000 & $0: 01: 39$ & $0: 00: 51$ & $0: 00: 59$ & $0: 00: 40$ & $48.3 \%$ & $33.0 \%$ & $22.6 \%$ \\
160000 & 160000 & $0: 03: 05$ & $0: 01: 31$ & $0: 01: 43$ & $0: 01: 09$ & $51.0 \%$ & $33.5 \%$ & $24.2 \%$ \\
200000 & 200000 & $0: 05: 03$ & $0: 02: 21$ & $0: 02: 43$ & $0: 01: 47$ & $53.5 \%$ & $34.2 \%$ & $24.0 \%$ \\
240000 & 240000 & $0: 07: 50$ & $0: 03: 23$ & $0: 03: 50$ & $0: 02: 30$ & $56.8 \%$ & $34.7 \%$ & $26.0 \%$ \\
280000 & 280000 & $0: 11: 11$ & $0: 04: 36$ & $0: 05: 13$ & $0: 03: 26$ & $58.8 \%$ & $34.2 \%$ & $25.6 \%$ \\
320000 & 320000 & $0: 15: 07$ & $0: 06: 00$ & $0: 06: 46$ & $0: 04: 25$ & $60.3 \%$ & $34.8 \%$ & $26.4 \%$ \\
360000 & 360000 & $0: 21: 25$ & $0: 07: 37$ & $0: 08: 44$ & $0: 05: 38$ & $64.5 \%$ & $35.4 \%$ & $25.9 \%$ \\
400000 & 400000 & $0: 24: 10$ & $0: 09: 22$ & $0: 10: 34$ & $0: 06: 55$ & $61.3 \%$ & $34.6 \%$ & $26.1 \%$ \\
\hline
\end{tabular}




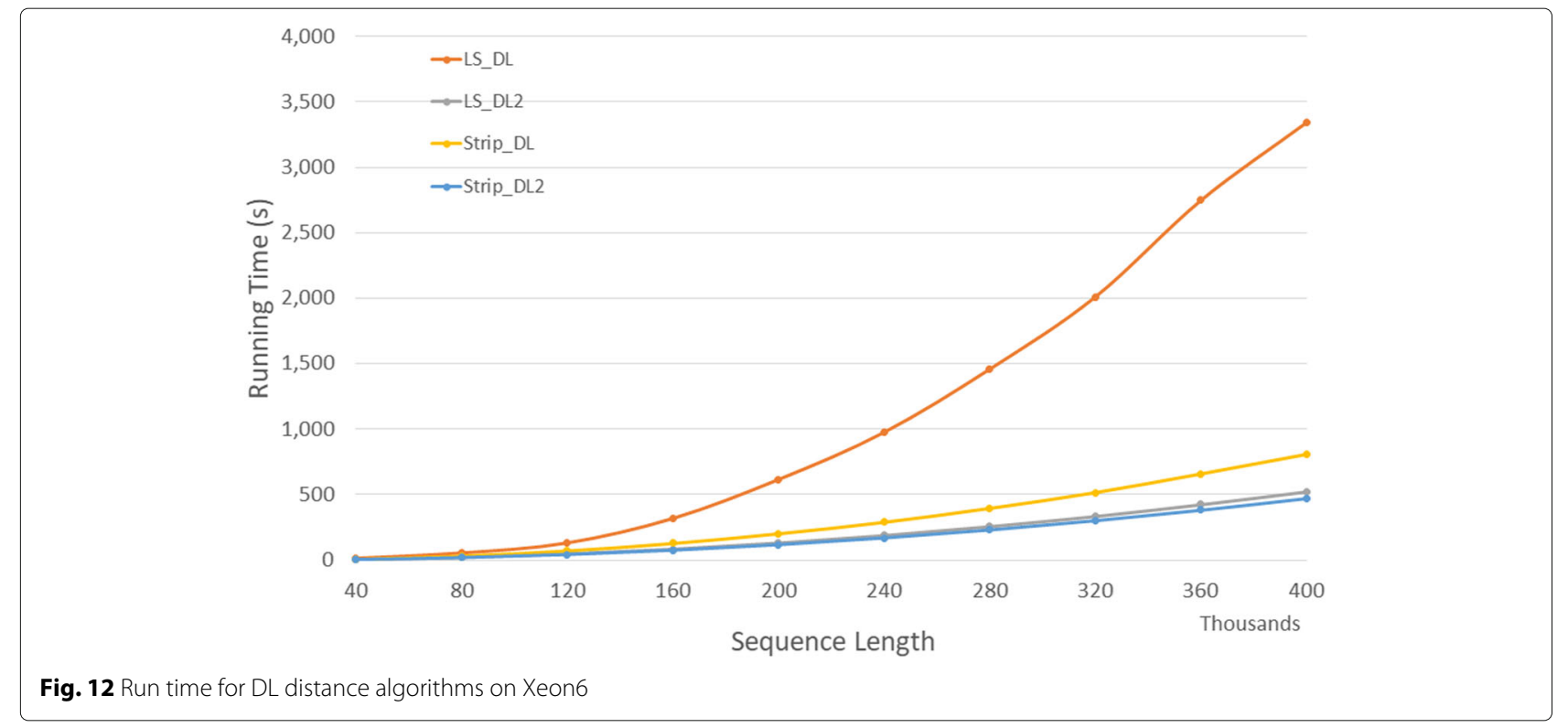

In a trace, unbalanced lines denote a substitution operation and balanced lines denote retaining the character of $A$. If $a_{i}$ has no line attached to it, $a_{i}$ is to be deleted and when $b_{j}$ has no attached line, it is to be inserted. When two balanced lines $\left(u_{1}, v_{1}\right)$ and $\left(u_{2}, v_{2}\right), u_{1}<u_{2}$ cross, $a_{u_{1}+1} \cdots a_{u_{2}-1}$ are to be deleted from $A$ making $a_{u_{1}}$ and $a_{u_{2}}$ adjacent, then $a_{u_{1}}$ and $a_{u_{2}}$ are to be transposed, and finally, $b_{v_{2}+1} \cdots b_{v_{1}-1}$ are to be inserted between the just transposed characters of $A$.

The edit sequence corresponding to the trace of Fig. 2 is delete $a_{2}$, transpose $a_{1}$ and $a_{3}$, substitute $b$ for $a_{4}$, insert $b_{4}=b$ and $b_{5}=e$, retain $a_{5}$. The cost of this edit sequence is 5 .

In [18], we used a divide-and-conquer strategy similar to that used by Hirschberg [20] to determine an optimal trace in $O(m n)$ time and $O(s \min \{m, n\}+n)$ space. In [18], we made a distinction between traces that have a center crossing and those that do not. A trace has a center crossing iff it contains two lines $\left(u_{1}, v_{1}\right)$ and $\left(u_{2}, v_{2}\right)$ such that $v_{2} \leq n / 2$ and $v_{1}>n / 2, u_{1}<u_{2}$, while satisfying (a) $a_{i} \neq a_{u_{1}}=b_{v_{1}}, u_{1}<i<u_{2}$ and (b) $b_{j} \neq b_{v_{2}}=a_{u_{2}}$, $v_{2}<j<v_{1}$. In words, $u_{1}$ is the last (i.e., rightmost)

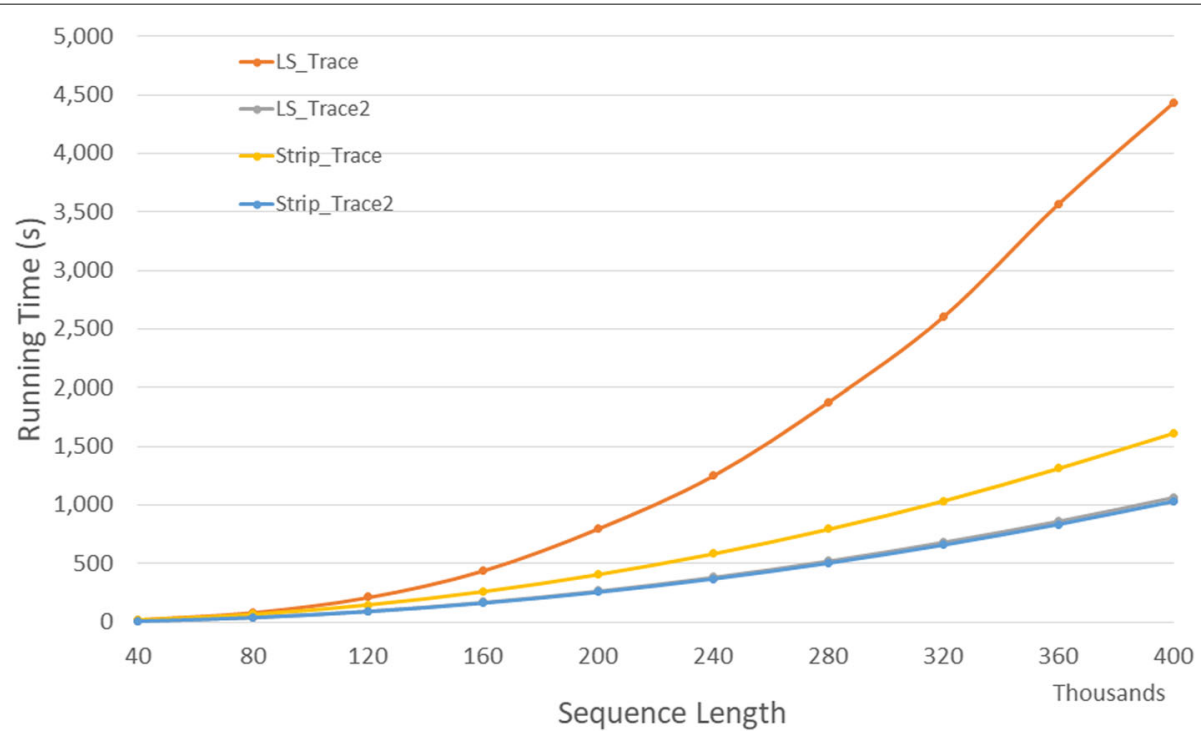

Fig. 13 Run time for DL trace algorithms on Xeon6 
occurrence of $b_{v_{1}}$ in $A$ that precedes position $u_{2}$ of $A$ and $v_{2}$ is the last occurrence of $a_{u_{2}}$ in $B$ that precedes position $v_{1}$ of $B$. (Figure 3).

In [18], we showed that the cost of an optimal trace $T$ is given by Eq. 7 when $T$ has no center crossing and by Eq. 8 when $T$ has a center crossing. Hence, the cost of $T$ is the smaller of these two costs.

$$
\operatorname{costNoCC}(T)=\min _{1 \leq i \leq m}\left\{H[i]+H^{\prime}[i+1]\right\}
$$

where $H[i]$ is the cost of an optimal trace for $A[1: i]$ and $B[1: n / 2]$ and $H^{\prime}[i+1]$ that for an optimal trace for $A[i+1: m]$ and $B[n / 2+1: n]$.

$$
\begin{aligned}
\operatorname{cost} C C(T)=\min \{ & H\left[u_{1}-1\right]\left[v_{2}-1\right]+H^{\prime}\left[u_{2}+1\right]\left[v_{1}+1\right] \\
& \left.+\left(u_{2}-u_{1}-1\right)+1+\left(v_{1}-v_{2}-1\right)\right\}
\end{aligned}
$$

where $H[i][j]$ is the cost of an optimal trace for $A[1: i]$ and $B[1][j]$ and $H^{\prime}[i][j]$ is that for an optimal trace for $A[i: m]$ and $B[j][n]$. For the $\min \{\}$, we try $1 \leq u_{1}<m$ and for each such $u_{1}$, we set $v_{1}$ to be the smallest $i>n / 2$ for which $b_{i}=a_{u_{1}}$. For each $u_{1}$ we examine all characters other than $a_{u_{1}}$ in the alphabet. For each such character $c$, $v_{2}$ is set to the largest $j \leq n / 2$ for which $b_{j}=c$ and $u_{2}$ is the smallest $i>u_{1}$ for which $a_{i}=c$.

Our new algorithms, LS_TRACE2 and Strip_TRACE2, are based on an adaptation of Eqs. 7 and 8 using Eq. 4.

\section{Algorithm LS_TRACE2}

Consider the case when the optimal trace has no center crossing. Let $R^{f}$ [ ] be the value of $R[]$ when $L S \_D L 2(B[1$ : $n / 2], A[1: m]$ ) terminates and let $R^{\prime f}$ [ ] be the value of $R[]$ when $L S \_D L 2(B[n: n / 2+1], A[m: 1])$ terminates. Let $R 1^{f}, R 1^{\prime f}, F R^{f}$, and $F R^{\prime f}$ be the corresponding final values for $R 1$ and $R F$. From Eq. 7 and $L D \_D L 2$, we obtain

$$
\begin{aligned}
\operatorname{costNoCC}(T) & =\min _{1 \leq i \leq m}\left\{H[i]+H^{\prime}[i+1]\right\} \\
& =\min _{1 \leq i \leq m}\left\{R^{f}[i]+R^{\prime f}[i+1]\right\}
\end{aligned}
$$

When $T$ has a center crossing $\left\{\left(u_{1}, v_{1}\right),\left(u_{2}, v_{2}\right)\right\}$, then it follows from Eq. 4 that either $u_{1}$ and $u_{2}$ are adjacent in $A$ or $v_{1}$ and $v_{2}$ are adjacent in $B$ (or both). When $v_{1}$ and $v_{2}$ are adjacent in $B$, then $v_{2}=\frac{n}{2}$ and $v_{1}=\frac{n}{2}+1$. Substituting into Eq. 8, we get

$$
\begin{aligned}
\operatorname{costCC}(T) & =\min \left\{H\left[u_{1}-1\right]\left[v_{2}-1\right]+H^{\prime}\left[u_{2}+1\right]\left[v_{1}+1\right]\right. \\
& \left.+\left(u_{2}-u_{1}-1\right)+1+\left(v_{1}-v_{2}-1\right)\right\} \\
& =\min \left\{H\left[u_{1}-1\right]\left[\frac{n}{2}-1\right]+H^{\prime}\left[u_{2}+1\right]\left[\frac{n}{2}+2\right]\right. \\
& \left.+\left(u_{2}-u_{1}\right)\right\} \\
& =\min \left\{R 1^{f}\left[u_{1}-1\right]+R 1^{\prime f}\left[u_{2}+1\right]+\left(u_{2}-u_{1}\right)\right\}
\end{aligned}
$$

When $u_{1}$ and $u_{2}$ are adjacent in $A$, then $u_{2}-u_{1}=1, v_{2}$ is the right most occurrence of $A\left[u_{2}\right]$ in $B$ that precedes position $\frac{n}{2}+1$ (i.e., $v_{2} \leq n / 2$ ) and $v_{1}$ is the left most occurrence of $A\left[u_{1}\right]$ in $B$ after position $\frac{n}{2}$ (i.e., $v_{1} \geq n / 2+1$ ). So, we have

$$
\begin{aligned}
\operatorname{costCC}(T) & =\min \left\{H\left[u_{1}-1\right]\left[v_{2}-1\right]+H^{\prime}\left[u_{2}+1\right]\left[v_{1}+1\right]\right. \\
& \left.+\left(u_{2}-u_{1}-1\right)+1+\left(v_{1}-v_{2}-1\right)\right\} \\
& =\min \left\{H\left[u_{1}-1\right]\left[v_{2}-1\right]+H^{\prime}\left[u_{2}+1\right]\left[v_{1}+1\right]\right. \\
& \left.+\left(v_{1}-v_{2}\right)\right\} \\
& =\min \left\{F R^{f}\left[u_{1}+1\right]+F R^{\prime f}\left[u_{2}-1\right]+\left(v_{1}-v_{2}\right)\right\}
\end{aligned}
$$

Algorithm LS_TRACE2 (Algorithm 2) provides the pseudocode for our linear space computation of an optimal trace. It assumes that $L S \_D L 2$ has been modified to return the arrays $R, R 1$ and $F R$.

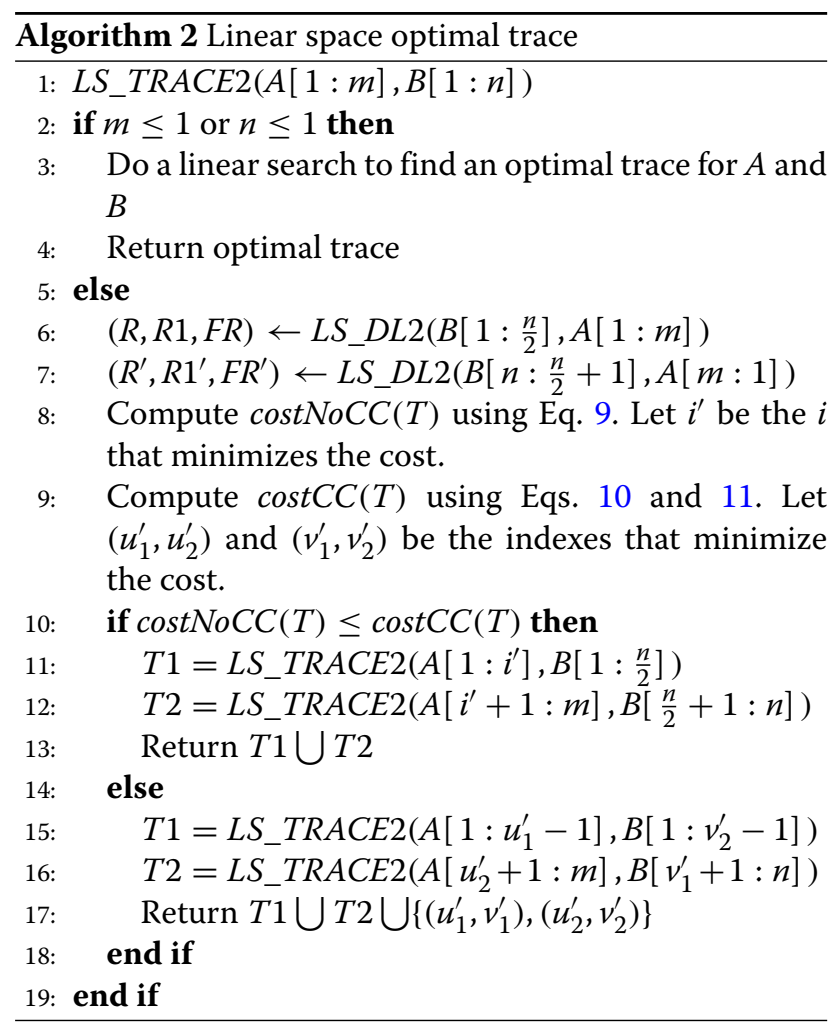

Using an analysis similar to that used by us in [18] for the analysis of $D L_{-} T R A C E$, we see that the time complexity of $D L_{-} T R A C E 2$ is $O(m n)$. The space required is the same as for $L S \_D L 2$. The number of cache misses is approximately twice that for $L S \_D L 2$ when invoked with strings of size $n$ and $m$. Hence, the cache miss count for $L S \_T R A C E 2$ is $\approx 6 m n / w$. 
Table 10 Run time in $h h: m m$ : ss for DL distance algorithms on Xeon6

\begin{tabular}{lllllllll}
\hline A & B & LS_DL & LS_DL2 & Strip_DL & Strip_DL2 & L vs L2 & S vs S2 & L2 vs S2 \\
\hline 40000 & 40000 & $0: 00: 14$ & $0: 00: 05$ & $0: 00: 08$ & $0: 00: 05$ & $63.2 \%$ & $41.7 \%$ & $9.8 \%$ \\
80000 & 80000 & $0: 00: 55$ & $0: 00: 21$ & $0: 00: 32$ & $0: 00: 19$ & $61.9 \%$ & $41.5 \%$ & $9.9 \%$ \\
120000 & 120000 & $0: 02: 12$ & $0: 00: 47$ & $0: 01: 13$ & $0: 00: 42$ & $64.3 \%$ & $41.5 \%$ & $9.7 \%$ \\
160000 & 160000 & $0: 05: 19$ & $0: 01: 24$ & $0: 02: 09$ & $0: 01: 15$ & $73.7 \%$ & $41.5 \%$ & $9.9 \%$ \\
200000 & 200000 & $0: 10: 16$ & $0: 02: 11$ & $0: 03: 23$ & $0: 01: 58$ & $78.7 \%$ & $41.8 \%$ & $9.9 \%$ \\
240000 & 240000 & $0: 16: 17$ & $0: 03: 09$ & $0: 04: 50$ & $0: 02: 50$ & $80.7 \%$ & $41.5 \%$ & $10.0 \%$ \\
280000 & 280000 & $0: 24: 19$ & $0: 04: 17$ & $0: 06: 36$ & $0: 03: 51$ & $82.4 \%$ & $41.7 \%$ & $10.1 \%$ \\
320000 & 320000 & $0: 33: 32$ & $0: 05: 35$ & $0: 08: 36$ & $0: 05: 02$ & $83.3 \%$ & $41.5 \%$ & $10.0 \%$ \\
360000 & 360000 & $0: 45: 50$ & $0: 07: 06$ & $0: 10: 58$ & $0: 06: 22$ & $84.5 \%$ & $42.0 \%$ & $10.2 \%$ \\
400000 & 400000 & $0: 55: 44$ & $0: 08: 44$ & $0: 13: 27$ & $0: 07: 52$ & $84.3 \%$ & $41.6 \%$ & $10.1 \%$ \\
\hline
\end{tabular}

\section{Strip trace algorithm Strip_TRACE2}

This algorithm differs from $L S \_T R A C E 2$ in that it uses a modified version of Strip_DL2 rather than a modified version of $L S \_D L 2$. The modified version of Strip_DL2 returns the arrays $C, C 1$ and $F C$ computed by Strip_DL2. The asymptotic time complexity of Strip_TRACE2 is also $O(m n)$ and it takes the same amount of space as does Strip_DL2. The number of cache misses is approximately twice that for Strip_DL2.

\section{Results}

We benchmarked the single-core algorithms $L S \_D L 2$, Strip_DL2,DL_TRACE2, and Strip_TRACE2 of this paper against the corresponding single-core algorithms developed by us in [18]. Using the parallelization techniques of [18], we obtained multi-core versions of our new algorithms. Their names are obtained by prefixing $P P_{-}$to the single-core name (e.g., $P P_{-} L S \_D L 2$ is the multi-core version of $\left.L S \_D L 2\right)$. The new multi-core versions also were benchmarked against the corresponding multi-core algorithms of [18].

\section{Platforms and test data}

The single-core algorithms were implemented using $\mathrm{C}$ and the multi-core ones using $\mathrm{C}$ and OpenMP. The relative performance of these algorithms was measured on the following platforms:

1. Intel Xeon CPU E5-2603 v2 Quad-Core processor $1.8 \mathrm{GHz}$ with $10 \mathrm{MB}$ cache.

2. Intel I7-x980 Six-Core processor $3.33 \mathrm{GHz}$ with 12MB LLC cache.

3. Intel Xeon CPU E5-2695 v2 2x12-Core processors 2.40GHz with $30 \mathrm{MB}$ cache.

For convenience, we will, at times, refer to these platforms as Xeon4, Xeon6, and Xeon24 (i.e., the number of cores is appended to the name Xeon).

All codes were compiled using the gcc compiler with the $\mathrm{O} 2$ option. On our Xeon4 platform, the benchmarking included a comparison of memory, cache misses, run time, and energy consumption. The cache miss count and the energy consumption was measured using the "perf" [21] software through the RAPL interface. For the Xeon6

Table 11 Run time in $h h$ : $m m$ : ss for DL trace algorithms on Xeon6

\begin{tabular}{|c|c|c|c|c|c|c|c|c|}
\hline$A$ & $B$ & LS_Trace & LS_Trace2 & Strip_Trace & Strip_Trace2 & L vs L2 & S vs S2 & L2 vs S2 \\
\hline 40000 & 40000 & 0:00:22 & 0:00:11 & 0:00:17 & 0:00:10 & $51.4 \%$ & $37.6 \%$ & $3.2 \%$ \\
\hline 80000 & 80000 & $0: 01: 24$ & 0:00:43 & 0:01:05 & 0:00:41 & $49.1 \%$ & $36.9 \%$ & $3.5 \%$ \\
\hline 120000 & 120000 & 0:03:33 & 0:01:36 & 0:02:26 & 0:01:33 & $54.9 \%$ & $36.8 \%$ & $3.6 \%$ \\
\hline 160000 & 160000 & 0:07:20 & $0: 02: 51$ & 0:04:20 & $0: 02: 44$ & $61.2 \%$ & $36.7 \%$ & $3.8 \%$ \\
\hline 200000 & 200000 & 0:13:19 & $0: 04: 27$ & 0:06:46 & 0:04:17 & $66.6 \%$ & $36.8 \%$ & $3.9 \%$ \\
\hline 240000 & 240000 & $0: 20: 51$ & $0: 06: 24$ & $0: 09: 43$ & $0: 06: 10$ & $69.3 \%$ & $36.6 \%$ & $3.8 \%$ \\
\hline 280000 & 280000 & $0: 31: 19$ & 0:08:43 & $0: 13: 14$ & 0:08:23 & $72.1 \%$ & $36.6 \%$ & $3.8 \%$ \\
\hline 320000 & 320000 & $0: 43: 24$ & $0: 11: 24$ & $0: 17: 16$ & $0: 10: 57$ & $73.7 \%$ & $36.6 \%$ & $3.9 \%$ \\
\hline 360000 & 360000 & $0: 59: 27$ & $0: 14: 23$ & $0: 21: 55$ & $0: 13: 52$ & $75.8 \%$ & $36.8 \%$ & $3.7 \%$ \\
\hline 400000 & 400000 & 1:13:51 & $0: 17: 47$ & $0: 26: 57$ & $0: 17: 07$ & $75.9 \%$ & $36.5 \%$ & $3.8 \%$ \\
\hline
\end{tabular}




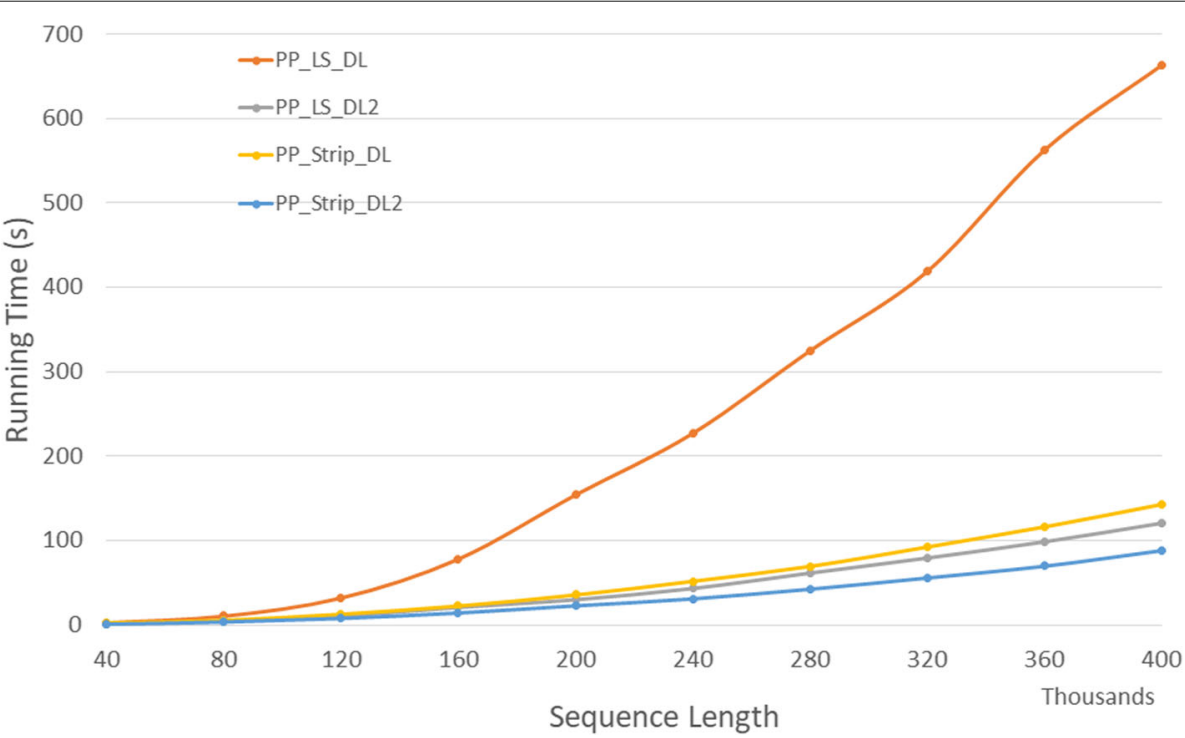

Fig. 14 Run time of parallel DL distance algorithms, in seconds, on Xeon6

and Xeon24 platforms only the run time was benchmarked.

For test data, we used randomly generated protein sequences as well as real protein sequences obtained from the Protein Data Bank [22] and DNA/RNA/protein sequences from the National Center for Biotechnology Information (NCBI) database [23]. The results for our randomly generated protein sequences were comparable to those for similarly sized sequences used from the two databases [22] and [23]. So, we present only the results for the random data sets here.

Xeon E5-2603 (Xeon4)

DL distance algorithms

Table 1 gives the memory required to process random protein sequences of length 400,000 using each of the single-core DL scoring algorithms considered in this paper. LS_DL takes 4.75 times the memory taken by LS_DL2 and LS_Strip takes 4.69 times the memory taken by LS_Strip2.

Figure 4 and Table 2 give the number of cache misses on our Xeon4 platform for randomly generated sequences of size between 40,000 and 400,000. The column of Table 2 labeled $L v s L 2$ gives the percentage reduction in cache misses achieved by $L S \_D L 2$ relative to $L S \_D L$, that labeled $S v s S 2$ gives this percentage for Strip_DL2 relative to Strip_DL, and that labeled $L 2 v S S 2$ gives this percentage for Strip_DL2 relative to $L S \_D L 2$. Strip_DL2 has the fewest cache misses. Strip_DL2 reduces cache misses by up to $91.9 \%$ relative to $L S \_D L 2$ and by up to $94.4 \%$ relative to Strip_DL.

Table 12 Run time of parallel DL distance algorithms, in $h h$ : $m m$ : ss, on Xeon6

\begin{tabular}{lllllllll}
\hline A & B & PP_LS_DL & PP_LS_DL2 & PP_Strip_DL & PP_Strip_DL2 & Lvs L2 & S vs S2 & L2 vs S2 \\
\hline 40000 & 40000 & $0: 00: 03$ & $0: 00: 01$ & $0: 00: 03$ & $0: 00: 01$ & $54.6 \%$ & $64.1 \%$ & $24.4 \%$ \\
80000 & 80000 & $0: 00: 11$ & $0: 00: 05$ & $0: 00: 06$ & $0: 00: 04$ & $54.2 \%$ & $37.4 \%$ & $27.2 \%$ \\
120000 & 120000 & $0: 00: 32$ & $0: 00: 11$ & $0: 00: 13$ & $0: 00: 08$ & $66.1 \%$ & $39.4 \%$ & $28.1 \%$ \\
160000 & 160000 & $0: 01: 18$ & $0: 00: 21$ & $0: 00: 23$ & $0: 00: 14$ & $72.8 \%$ & $36.4 \%$ & $32.5 \%$ \\
200000 & 200000 & $0: 02: 34$ & $0: 00: 30$ & $0: 00: 36$ & $0: 00: 23$ & $80.3 \%$ & $35.9 \%$ & $24.4 \%$ \\
240000 & 240000 & $0: 03: 47$ & $0: 00: 44$ & $0: 00: 52$ & $0: 00: 31$ & $80.8 \%$ & $39.8 \%$ & $28.5 \%$ \\
280000 & 280000 & $0: 05: 25$ & $0: 01: 02$ & $0: 01: 09$ & $0: 00: 43$ & $80.9 \%$ & $38.4 \%$ & $31.0 \%$ \\
320000 & 320000 & $0: 06: 59$ & $0: 01: 20$ & $0: 01: 32$ & $0: 00: 56$ & $80.9 \%$ & $39.2 \%$ & $29.6 \%$ \\
360000 & 360000 & $0: 09: 23$ & $0: 01: 39$ & $0: 01: 56$ & $0: 01: 10$ & $82.4 \%$ & $39.6 \%$ & $29.2 \%$ \\
400000 & 400000 & $0: 11: 03$ & $0: 02: 01$ & $0: 02: 23$ & $0: 01: 29$ & $81.7 \%$ & $37.8 \%$ & $26.7 \%$ \\
\hline
\end{tabular}




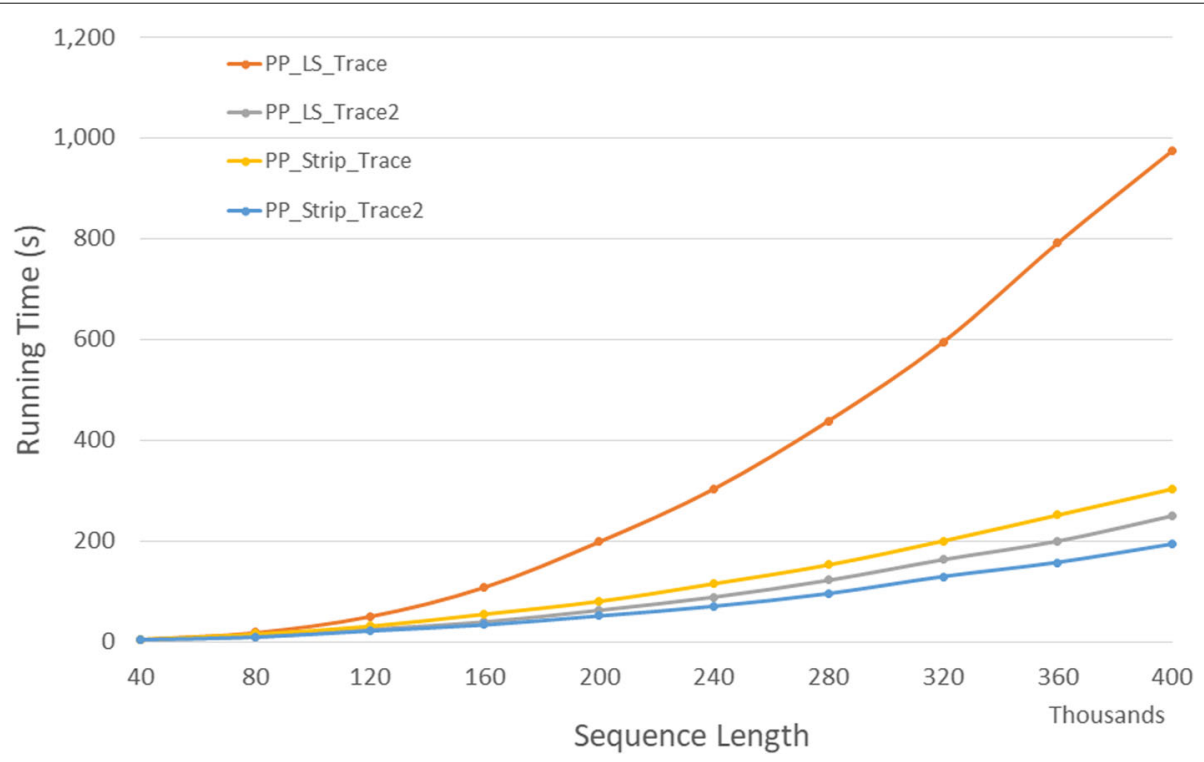

Fig. 15 Run time of parallel DL trace algorithms, in seconds, on Xeon6

Figure 5 and Table 3 give the run times on our Xeon 4 platform for our random data set. In the figure, the time is in seconds while in the table, the time is given using the format $h h: m m: s s$. The table also gives the percentage reduction in run time.

As can be seen, on our Xeon4 platform, Strip_DL2 is the fastest followed by $L S \_D L 2$, Strip_DL, and $L S \_D L$. Strip_DL2 reduces run time by up to $15.9 \%$ relative to $L S \_D L 2$ and by up to $40.1 \%$ relative to Strip_DL.

Figure 6 and Table 4 give the CPU and cache energy consumed, in joules, on our Xeon4 platform. On our data sets, Strip_DL2 required up to $17.6 \%$ less CPU and cache energy than $L S \_D L 2$ and up to $40.0 \%$ less than Strip_DL. It is interesting to note that the energy reduction is comparable to the reduction in run time suggesting a close relationship between run time and energy consumption for this application.

\section{DL trace algorithms}

Figure 7 and Table 5 give the number of cache misses for the trace algorithms on our Xeon4 platform for randomly generated sequences of size between 40,000 and 400,000. The column of Table 5 labeled LvsL2 gives the percentage reduction in cache misses achieved by $L S \_T r a c e 2$ relative to $L S \_T r a c e$, that labeled SvsS2 gives this percentage Strip_Trace 2 relative to Strip_Trace, and that labeled $L 2 v s S 2$ gives this percentage Strip_Trace 2 relative to $L S \_T r a c e 2$. Strip_Trace2 has the fewest cache

Table 13 Run time of parallel DL trace algorithms, in $h h: m m$ : ss, on Xeon6

\begin{tabular}{|c|c|c|c|c|c|c|c|c|}
\hline A & B & PP_LS_Trace & PP_LS_Trace2 & PP_Strip_Trace & PP_Strip_Trace2 & L vs L2 & S vs S2 & L2 vs S2 \\
\hline 40000 & 40000 & 0:00:05 & 0:00:05 & 0:00:05 & 0:00:04 & $10.3 \%$ & $20.6 \%$ & $14.3 \%$ \\
\hline 80000 & 80000 & 0:00:19 & 0:00:10 & 0:00:16 & 0:00:09 & $44.8 \%$ & $41.8 \%$ & $11.0 \%$ \\
\hline 120000 & 120000 & 0:00:51 & $0: 00: 25$ & $0: 00: 31$ & $0: 00: 21$ & $51.0 \%$ & $31.8 \%$ & $13.8 \%$ \\
\hline 160000 & 160000 & 0:01:49 & 0:00:40 & 0:00:56 & 0:00:33 & $63.3 \%$ & $39.9 \%$ & $16.3 \%$ \\
\hline 200000 & 200000 & 0:03:19 & 0:01:03 & $0: 01: 21$ & $0: 00: 51$ & $68.4 \%$ & $36.7 \%$ & $18.7 \%$ \\
\hline 240000 & 240000 & 0:05:04 & 0:01:29 & 0:01:56 & 0:01:10 & $70.7 \%$ & $39.2 \%$ & $21.0 \%$ \\
\hline 280000 & 280000 & 0:07:19 & 0:02:02 & $0: 02: 33$ & 0:01:36 & $72.1 \%$ & $37.6 \%$ & $21.9 \%$ \\
\hline 320000 & 320000 & 0:09:55 & 0:02:43 & 0:03:21 & 0:02:09 & $72.5 \%$ & $35.5 \%$ & $20.8 \%$ \\
\hline 360000 & 360000 & 0:13:11 & 0:03:20 & $0: 04: 12$ & 0:02:38 & $74.7 \%$ & $37.4 \%$ & $21.3 \%$ \\
\hline 400000 & 400000 & $0: 16: 14$ & 0:04:10 & 0:05:03 & 0:03:14 & $74.3 \%$ & $36.1 \%$ & $22.5 \%$ \\
\hline
\end{tabular}


Table 14 Run time in $h h: m m$ : ss for DL distance algorithms on Xeon24

\begin{tabular}{lllllllll}
\hline A & B & LS_DL & LS_DL2 & Strip_DL & Strip_DL2 & L vs L2 & S vs S2 & L2 vs S2 \\
\hline 40000 & 40000 & $0: 00: 14$ & $0: 00: 06$ & $0: 00: 10$ & $0: 00: 05$ & $58.0 \%$ & $50.6 \%$ & $13.8 \%$ \\
80000 & 80000 & $0: 00: 53$ & $0: 00: 22$ & $0: 00: 41$ & $0: 00: 20$ & $58.1 \%$ & $51.6 \%$ & $11.9 \%$ \\
120000 & 120000 & $0: 02: 01$ & $0: 00: 57$ & $0: 01: 31$ & $0: 00: 49$ & $53.1 \%$ & $46.5 \%$ & $13.5 \%$ \\
160000 & 160000 & $0: 03: 34$ & $0: 01: 28$ & $0: 02: 42$ & $0: 01: 18$ & $58.8 \%$ & $51.9 \%$ & $11.6 \%$ \\
200000 & 200000 & $0: 06: 08$ & $0: 02: 19$ & $0: 04: 15$ & $0: 02: 01$ & $62.1 \%$ & $52.4 \%$ & $12.9 \%$ \\
240000 & 240000 & $0: 08: 05$ & $0: 03: 16$ & $0: 06: 05$ & $0: 02: 56$ & $59.6 \%$ & $51.8 \%$ & $10.4 \%$ \\
280000 & 280000 & $0: 11: 32$ & $0: 04: 27$ & $0: 08: 20$ & $0: 03: 59$ & $61.5 \%$ & $52.1 \%$ & $10.2 \%$ \\
320000 & 320000 & $0: 15: 55$ & $0: 05: 52$ & $0: 10: 58$ & $0: 05: 13$ & $63.1 \%$ & $52.4 \%$ & $10.9 \%$ \\
360000 & 360000 & $0: 26: 41$ & $0: 07: 24$ & $0: 15: 11$ & $0: 06: 38$ & $72.2 \%$ & $56.4 \%$ & $10.5 \%$ \\
400000 & 400000 & $0: 30: 11$ & $0: 09: 02$ & $0: 17: 15$ & $0: 08: 06$ & $70.1 \%$ & $53.0 \%$ & $10.3 \%$ \\
\hline
\end{tabular}

misses. Strip_Trace2 reduces cache misses by up to $89.1 \%$ relative to $L S \_$Trace 2 and by up to $95.4 \%$ relative to Strip_Trace.

Figure 8 and Table 6 give the run times on our Xeon 4 platform for our random data set. The table also gives the percentage reduction in run time. Strip_Trace 2 is the fastest followed by $L S \_T r a c e 2$, Strip_Trace, and $L S \_T r a c e$ (in this order). Strip_Trace 2 reduces run time by up to $4.3 \%$ relative to $L S \_T r a c e 2$ and by up to $37.8 \%$ relative to Strip_Trace.

Figure 9 and Table 7 give the CPU and cache energy consumed, in joules, Strip_Trace 2 required up to $6.3 \%$ less CPU and cache energy than LS_Trace2 and up to $37.9 \%$ less than Strip_Trace.

\section{Parallel algorithms}

Figure 10 and Table 8 give the run times for our parallel DL distance algorithms on our Xeon 4 platform. $P P \_L S \_D L 2$ is up to $61.2 \%$ faster than $P P_{-} L S \_D L$ and $P P_{-} S t r i p \_D L 2$ is up to $40.6 \%$ faster than PP_Strip_DL. Also, PP_LS_DL2 and PP_Strip_DL2 achieve a speedup of up to 3.15 and 3.98 compared to the corresponding single-core algorithms on a four-core machine, respectively.

Figure 11 and Table 9 give the run times for our parallel DL trace algorithms on our Xeon4 platform. PP_LS_Trace 2 is up to $64.5 \%$ faster than PP_LS_Trace and PP_Strip_Trace 2 is up to $35.4 \%$ faster than PP_Strip_Trace. Also, PP_LS_Trace 2 and PP_Strip_Trace 2 achieves a speedup up to 2.94 and 3.83 compared to the corresponding single-core algorithms, respectively.

\section{7-x980 (Xeon6)}

DL distance algorithms

Figure 12 and Table 10 give the run times of our singlecore distance algorithms on our Xeon6 platform. As can

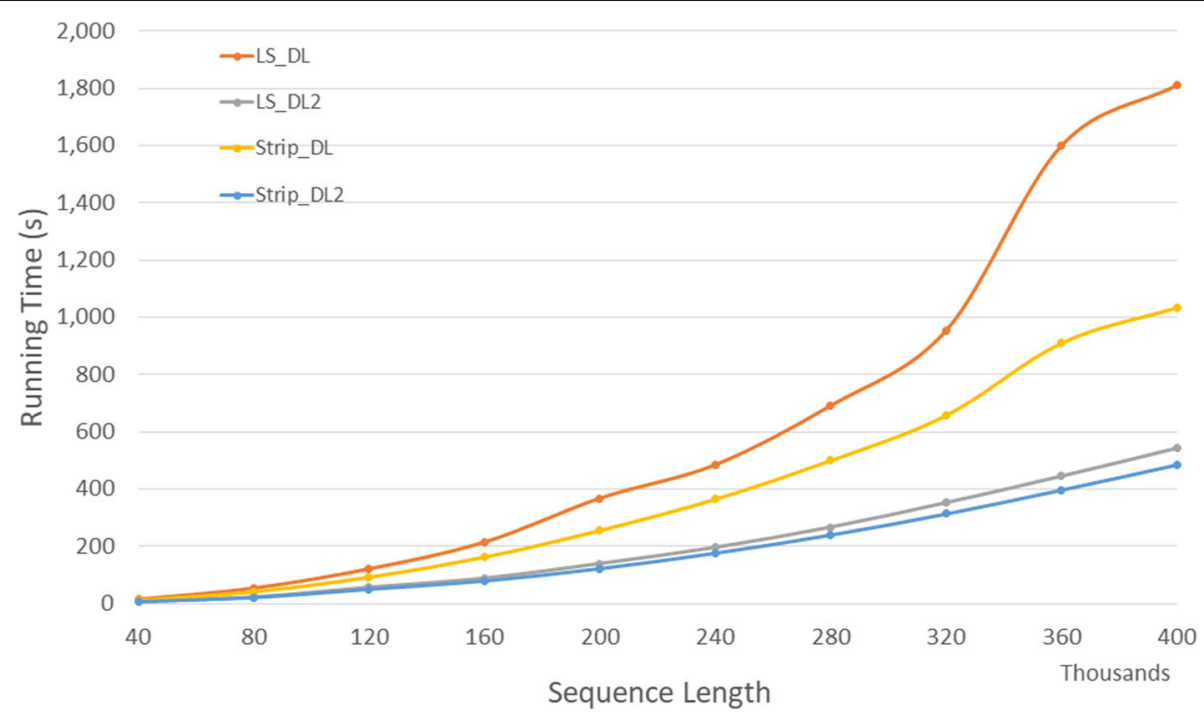

Fig. 16 Run time for DL distance algorithms on Xeon24 


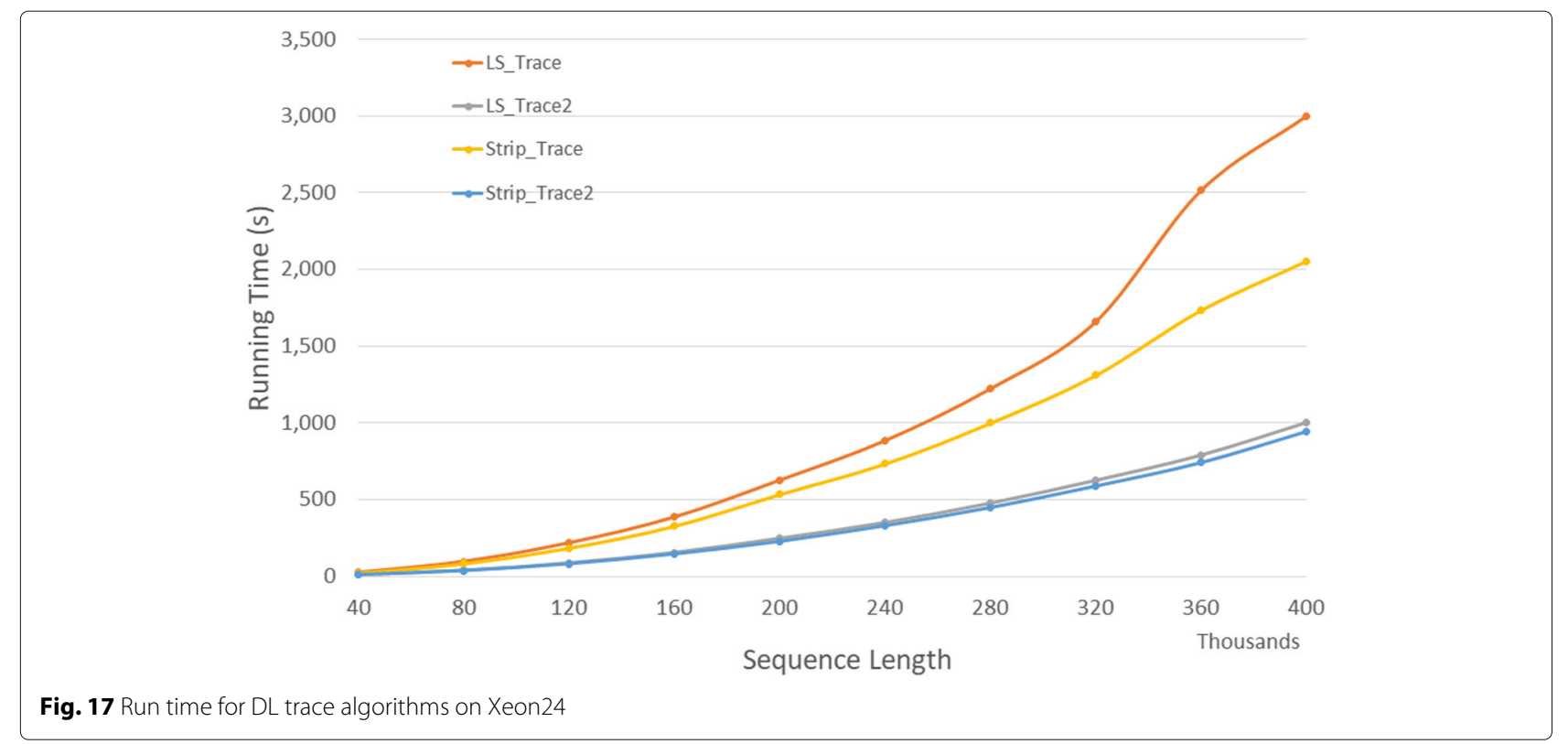

be seen, Strip_DL2 is the fastest followed by $L S \_D L 2$, Strip_DL, and $L S \_D L$ (in this order). Strip_DL2 reduces run time by up to $10.2 \%$ relative to $L S \_D L 2$ and by up to $42.0 \%$ relative to Strip_DL.

\section{DL trace algorithms}

Figure 13 and Table 11 give the run times of our single-core trace algorithms on our Xeon6 platform. Strip_Trace 2 is the fastest followed by LS_Trace2, Strip_Trace, and LS_Trace (in this order). Strip_Trace 2 reduces run time by up to $3.9 \%$ relative to $L S \_T r a c e 2$ and by up to $37.6 \%$ relative to Strip_Trace.

\section{Parallel algorithms}

Figure 14 and Table 12 give the run times for our parallel DL distance algorithms on our Xeon6 platform. PP_LS_DL2 is up to $82.4 \%$ faster than PP_LS_DL and PP_Strip_DL2 is up to $39.8 \%$ faster than PP_Strip_DL.
Also, PP_LS_DL2 and PP_Strip_DL2 achieves a speedup up to 4.32 and 5.44 compared to the corresponding single core algorithms on a six core machine, respectively.

Figure 15 and Table 13 give the run times for our parallel DL trace algorithms on our Xeon6 platform. PP_LS_Trace 2 is up to $74.7 \%$ faster than PP_LS_Trace and PP_Strip_Trace 2 is up to $41.8 \%$ faster than PP_Strip_Trace. Also, PP_LS_Trace 2 and PP_Strip_Trace 2 achieves a speedup up to 4.32 and 5.30 compared to the corresponding single-core algorithms, respectively.

\section{Xeon E5-2695 (Xeon24)}

DL distance algorithms

Figure 16 and Table 14 give the run times of our single-core distance algorithms on our Xeon24 platform. Strip_DL2 is the fastest followed by $L S \_D L 2$, Strip_DL,

Table 15 Run time in $h h: m m$ : ss for DL trace algorithms on Xeon24

\begin{tabular}{lllllllll}
\hline A & B & LS_Trace & LS_Trace2 & Strip_Trace & Strip_Trace2 & Lvs L2 & S vs S2 & L2 vs S2 \\
\hline 40000 & 40000 & $0: 00: 25$ & $0: 00: 12$ & $0: 00: 21$ & $0: 00: 11$ & $52.7 \%$ & $49.7 \%$ & $9.4 \%$ \\
80000 & 80000 & $0: 01: 36$ & $0: 00: 40$ & $0: 01: 23$ & $0: 00: 37$ & $58.5 \%$ & $55.4 \%$ & $7.1 \%$ \\
120000 & 120000 & $0: 03: 38$ & $0: 01: 28$ & $0: 03: 04$ & $0: 01: 22$ & $59.5 \%$ & $55.5 \%$ & $7.0 \%$ \\
160000 & 160000 & $0: 06: 26$ & $0: 02: 36$ & $0: 05: 27$ & $0: 02: 26$ & $59.5 \%$ & $55.4 \%$ & $6.6 \%$ \\
200000 & 200000 & $0: 10: 26$ & $0: 04: 08$ & $0: 08: 54$ & $0: 03: 47$ & $60.3 \%$ & $57.4 \%$ & $8.4 \%$ \\
240000 & 240000 & $0: 14: 44$ & $0: 05: 51$ & $0: 12: 14$ & $0: 05: 30$ & $60.3 \%$ & $55.0 \%$ & $6.0 \%$ \\
280000 & 280000 & $0: 20: 22$ & $0: 07: 57$ & $0: 16: 39$ & $0: 07: 28$ & $61.0 \%$ & $55.2 \%$ & $6.1 \%$ \\
320000 & 320000 & $0: 27: 39$ & $0: 10: 26$ & $0: 21: 51$ & $0: 09: 48$ & $62.3 \%$ & $55.1 \%$ & $6.1 \%$ \\
360000 & 360000 & $0: 41: 56$ & $0: 13: 10$ & $0: 28: 55$ & $0: 12: 21$ & $68.6 \%$ & $57.3 \%$ & $6.2 \%$ \\
400000 & 400000 & $0: 50: 01$ & $0: 16: 42$ & $0: 34: 16$ & $0: 15: 43$ & $66.6 \%$ & $54.1 \%$ & $5.9 \%$ \\
\hline
\end{tabular}




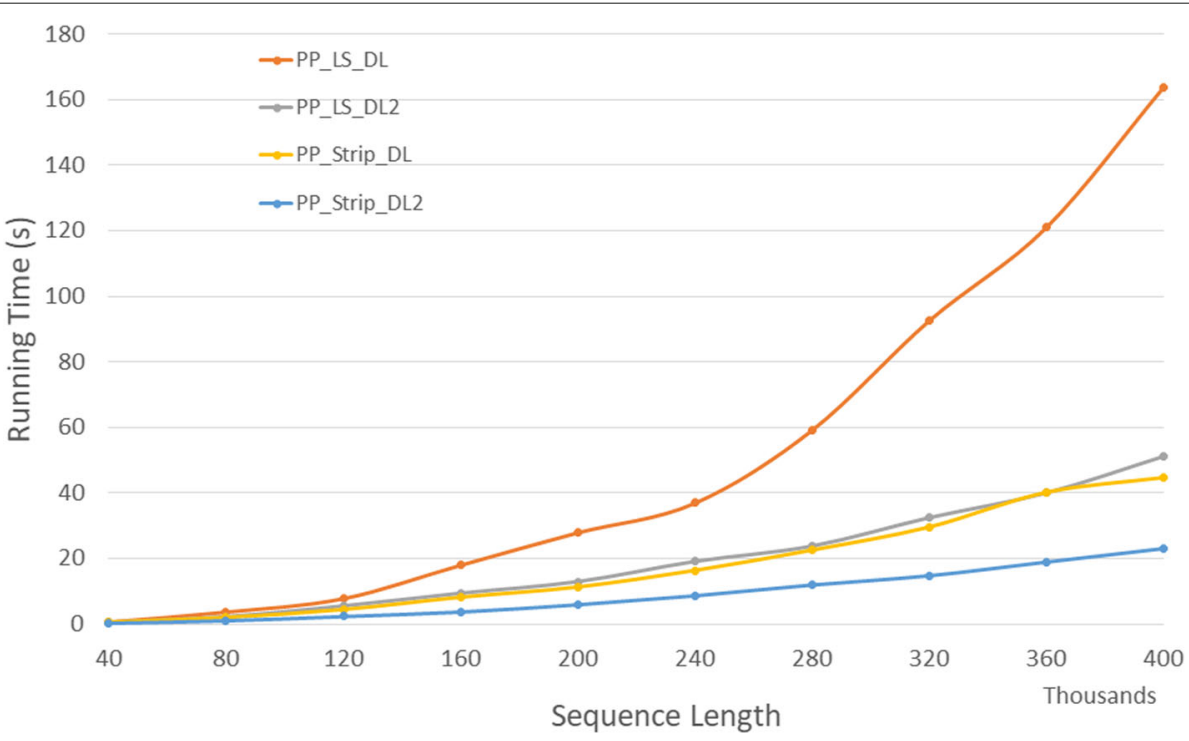

Fig. 18 Run time of parallel $\mathrm{DL}$ distance algorithms, in seconds, on Xeon24

and $L S \_D L$ (in this order). Strip_DL2 reduces run time by up to $13.8 \%$ relative to $L S \_D L 2$ and by up to $56.4 \%$ relative to Strip_DL.

\section{DL trace algorithms}

Figure 17 and Table 15 give the run times of our single-core trace algorithms on our Xeon24 platform. Strip_Trace 2 is the fastest followed by LS_Trace2, Strip_Trace, and LS_Trace (in this order). Strip_Trace2 reduces run time by up to $9.4 \%$ relative to $L S \_$Trace 2 and by up to $57.4 \%$ relative to Strip_Trace.

\section{Parallel algorithms}

Figure 18 and Table 16 give the run times for our parallel DL distance algorithms on our Xeon24 platform.
PP_LS_DL2 is up to $68.7 \%$ faster than $P P_{-} L S_{-} D L$ and PP_Strip_DL2 is up to $54.6 \%$ faster than PP_Strip_DL. Also, $P P_{-} L S \_D L 2$ and PP_Strip_DL2 achieves a speedup up to 11.2 and 21.36 compared to the corresponding single core algorithms on a twelve-four core machine, respectively.

Figure 19 and Table 17 give the run times for our parallel DL trace algorithms on our Xeon24 platform. PP_LS_Trace 2 is up to $58.3 \%$ faster than PP_LS_Trace and PP_Strip_Trace 2 is up to $59.3 \%$ faster than PP_Strip_Trace. Also, PP_LS_Trace 2 and PP_Strip_Trace 2 achieves a speedup up to 9.65 and 16.4 compared to the corresponding single-core algorithms, respectively.

Table 16 Run time of parallel DL distance algorithms, in $h h: m m$ : ss, on Xeon24

\begin{tabular}{lllllllll}
\hline A & B & PP_LS_DL & PP_LS_DL2 & PP_Strip_DL & PP_Strip_DL2 & L vs L2 & S vs S2 & L2 vs S2 \\
\hline 40000 & 40000 & $0: 00: 01$ & $0: 00: 01$ & $0: 00: 01$ & $0: 00: 00$ & $30.9 \%$ & $48.6 \%$ & $41.6 \%$ \\
80000 & 80000 & $0: 00: 04$ & $0: 00: 02$ & $0: 00: 02$ & $0: 00: 01$ & $41.8 \%$ & $47.2 \%$ & $51.4 \%$ \\
120000 & 120000 & $0: 00: 08$ & $0: 00: 06$ & $0: 00: 04$ & $0: 00: 02$ & $30.2 \%$ & $47.4 \%$ & $57.4 \%$ \\
160000 & 160000 & $0: 00: 18$ & $0: 00: 09$ & $0: 00: 08$ & $0: 00: 04$ & $48.1 \%$ & $54.6 \%$ & $60.3 \%$ \\
200000 & 200000 & $0: 00: 28$ & $0: 00: 13$ & $0: 00: 11$ & $0: 00: 06$ & $53.8 \%$ & $47.6 \%$ & $54.2 \%$ \\
240000 & 240000 & $0: 00: 37$ & $0: 00: 19$ & $0: 00: 16$ & $0: 00: 09$ & $48.4 \%$ & $47.3 \%$ & $54.8 \%$ \\
280000 & 280000 & $0: 00: 59$ & $0: 00: 24$ & $0: 00: 23$ & $0: 00: 12$ & $59.8 \%$ & $47.2 \%$ & $50.0 \%$ \\
320000 & 320000 & $0: 01: 33$ & $0: 00: 32$ & $0: 00: 30$ & $0: 00: 15$ & $65.0 \%$ & $50.3 \%$ & $54.8 \%$ \\
360000 & 360000 & $0: 02: 01$ & $0: 00: 40$ & $0: 00: 40$ & $0: 00: 19$ & $66.9 \%$ & $53.1 \%$ & $53.0 \%$ \\
400000 & 400000 & $0: 02: 44$ & $0: 00: 51$ & $0: 00: 45$ & $0: 00: 23$ & $68.7 \%$ & $48.7 \%$ & $55.3 \%$ \\
\hline
\end{tabular}




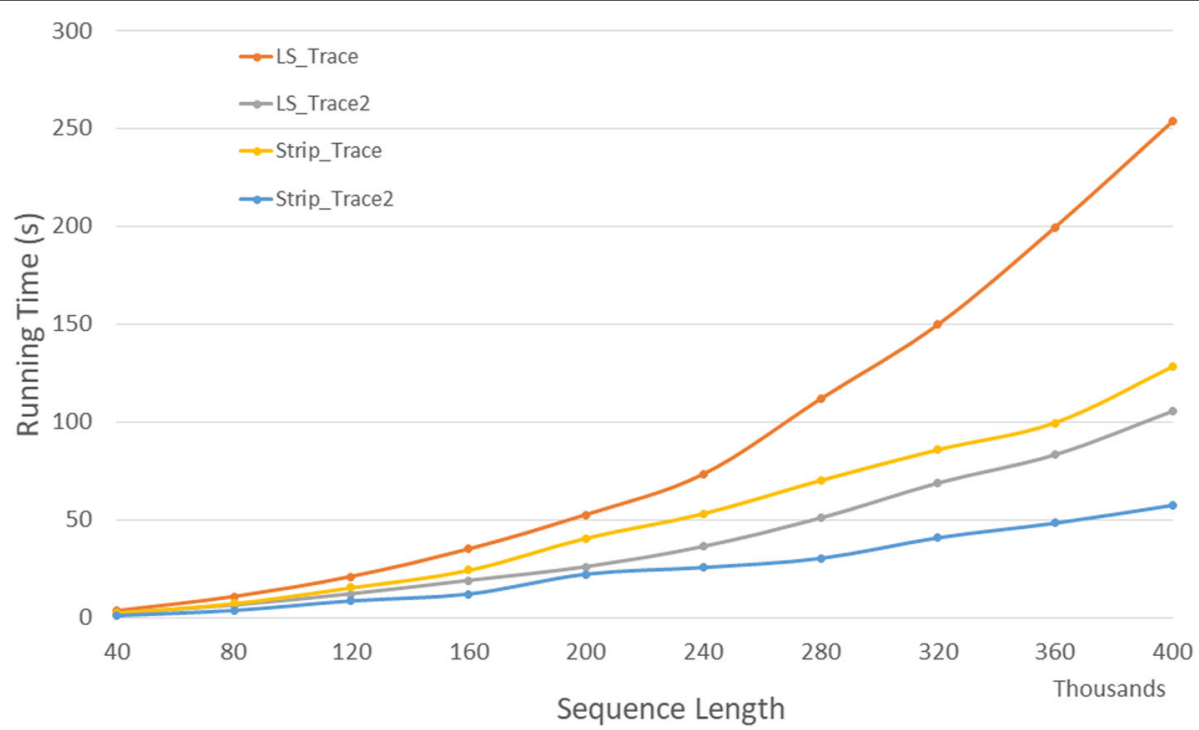

Fig. 19 Run time of parallel DL trace algorithms, in seconds, on Xeon24

\section{Discussion}

We have developed linear space algorithms to compute the DL distance between two strings and also to determine an optimal trace (edit sequence). Besides using less space than the space efficient algorithms of [18], these algorithms are also faster. Significant run-time improvement (relative to known algorithms) was seen for our new algorithms on all three of our experimental platforms.

\section{Conclusion}

On all platforms, the linear-space cache-efficient algorithms Strip_DL2 and Strip_TRACE2 were the bestperforming single-core algorithms to determine the DL distance and optimal trace, respectively. Strip_DL2 reduced run time by as much as $56.4 \%$ relative to the Strip_DL and Strip_TRACE2 reduced run time by as much as $57.4 \%$ relative to the Strip_Trace. Our multi-core algorithms reduced the run time by up to $59.3 \%$ compared to the best previously known multi-core algorithms.

The linear space string correction algorithm developed in this paper requires $2 S \leq I+D \leq 2 T$, where $S$, $I, D$ and $T$ are, respectively, the cost of a substitution, insertion, deletion and transposition. As noted earlier, this requirement is met by the DL distance as for this metric, $S=I=D=T=1$. When only $I+D \leq 2 T$ is satisfied, the more general algorithm ([18]) that runs in $O(m n)$ time and uses $O(s * \min \{m, n\}+m+n)$ space, where $s$ is the size of alphabet comprising the strings, may be used.

Table 17 Run time of parallel DL trace algorithms, in $h h: m m: s s$, on Xeon24

\begin{tabular}{|c|c|c|c|c|c|c|c|c|}
\hline A & B & PP_LS_Trace & PP_LS_Trace2 & PP_Strip_Trace & PP_Strip_Trace2 & L vs L2 & S vs S2 & L2 vs S2 \\
\hline 40000 & 40000 & 0:00:03 & 0:00:02 & 0:00:02 & 0:00:01 & $38.0 \%$ & $59.3 \%$ & $53.3 \%$ \\
\hline 80000 & 80000 & 0:00:11 & 0:00:06 & 0:00:07 & 0:00:04 & $40.3 \%$ & $49.2 \%$ & $42.5 \%$ \\
\hline 120000 & 120000 & $0: 00: 21$ & 0:00:12 & 0:00:15 & 0:00:09 & $42.3 \%$ & $44.1 \%$ & $29.2 \%$ \\
\hline 160000 & 160000 & 0:00:35 & 0:00:19 & $0: 00: 24$ & 0:00:12 & $45.8 \%$ & $50.2 \%$ & $36.5 \%$ \\
\hline 200000 & 200000 & 0:00:53 & $0: 00: 26$ & $0: 00: 41$ & $0: 00: 22$ & $50.6 \%$ & $45.2 \%$ & $14.2 \%$ \\
\hline 240000 & 240000 & $0: 01: 13$ & 0:00:36 & $0: 00: 53$ & $0: 00: 26$ & $50.4 \%$ & $51.7 \%$ & $29.4 \%$ \\
\hline 280000 & 280000 & 0:01:52 & 0:00:51 & 0:01:10 & 0:00:30 & $54.4 \%$ & $56.7 \%$ & $40.4 \%$ \\
\hline 320000 & 320000 & 0:02:30 & 0:01:09 & $0: 01: 26$ & $0: 00: 41$ & $54.0 \%$ & $52.3 \%$ & $40.6 \%$ \\
\hline 360000 & 360000 & 0:03:20 & $0: 01: 23$ & 0:01:40 & 0:00:48 & $58.2 \%$ & $51.4 \%$ & $41.9 \%$ \\
\hline 400000 & 400000 & $0: 04: 14$ & 0:01:46 & 0:02:08 & 0:00:58 & $58.3 \%$ & $55.1 \%$ & $45.5 \%$ \\
\hline
\end{tabular}




\section{Abbreviations}

CPU: Central Processing Unit; DL: Damerau-Levenshtein; DNA:

DeoxyriboNucleic acid; LLC: Last level cache; LRU: Least recently used; NCBI:

National Center for Biotechnology Information; PDB: Protein Data Bank; RNA:

RiboNucleic acid

\section{Acknowledgments}

The authors also would like to thank the anonymous reviewers for their valuable comments and suggestions.

\section{About this supplement}

This article has been published as part of BMC Bioinformatics Volume 21 Supplement 1, 2020: Selected articles from the 8th IEEE International Conference on Computational Advances in Bio and medical Sciences (ICCABS 2018): bioinformatics. The full contents of the supplement are available online at https://bmcbioinformatics.biomedcentral.com/articles/supplements/volume21-supplement-1.

\section{Authors' contributions}

CZ and SS developed the new linear space cache efficient DL distance algorithms, did theoretical analysis and the experimental results analysis, and wrote the manuscript. CZ programmed the algorithms and ran the benchmark tests. All authors have read and approved the final manuscript.

\section{Funding}

Publication of this supplement was funded, in part, by the National Science Foundation under award NSF 1447711. Publication costs were funded by the University of Florida.

\section{Availability of data and materials}

Data sharing is not applicable to this article as all test data are randomly generated protein sequences.

\section{Ethics approval and consent to participate}

Not applicable.

\section{Consent for publication}

Not applicable.

\section{Competing interests}

The authors declare that they have no competing interests.

Received: 20 October 2019 Accepted: 30 October 2019 Published: 9 December 2020

\section{References}

1. Brill E, Moore RC. An improved error model for noisy channel spelling correction. In: Proceedings of the 38th Annual Meeting on Association for Computational Linguistics. Stroudsburg: Association for Computational Linguistics; 2000. p. 286-93.

2. Bard GV. Spelling-error tolerant, order-independent pass-phrases via the Damerau-Levenshtein string-edit distance metric. In: Proceedings of the Fifth Australasian Symposium on ACSW Frontiers - Volume 68. ACSW '07. Darlinghurst: Australian Computer Society, Inc.; 2007. p. 117-24.

3. Li M, Zhang Y, Zhu M, Zhou M. Exploring distributional similarity based models for query spelling correction. In: Proceedings of the $21 \mathrm{st}$ International Conference on Computational Linguistics and the 44th Annual Meeting of the Association for Computational Linguistics. ACL-44. Stroudsburg: Association for Computational Linguistics; 2006. p. 1025-32.

4. Cai X, Zhang XC, Joshi B, Johnson R. Touching from a distance: Website fingerprinting attacks and defenses. In: Proceedings of the 2012 ACM Conference on Computer and Communications Security. New York: ACM; 2012. p. 605-16.

5. Faloutsos C, Megalooikonomou V. On data mining, compression, and kolmogorov complexity. Data Min Knowl Discov. 2007;15(1):3-20.

6. Majorek KA, Dunin-Horkawicz S, Steczkiewicz K, Muszewska A, Nowotny M, Ginalski K, Bujnicki JM. The RNase H-like superfamily: new members, comparative structural analysis and evolutionary classification. In: Nucleic Acids Research, vol. 42. Oxford, United Kingdom: Oxford University Press; 2014. p. 4160-4179.
7. Bischof J, Ibrahim SM. bcRep: R package for comprehensive analysis of B cell receptor repertoire data. PLoS ONE. 2016;11(8):1-15.

8. Pomorova O, Savenko O, Lysenko S, Nicheporuk A. Information and Communication Technologies in Education, Research, and Industrial Applications. In: 12th Internal conference. Kyiv: Springer; 2016. http:// ceur-ws.org/Nol-1614/.

9. Biswas AK, Gao JX. PR2S2Clust: patched rna-seq read segments' structure-oriented clustering. J Bioinforma Comput Biol. 2016;14(05): 1650027.

10. Pop PG. In-memory dedicated dot-plot analysis for DNA repeats detection. In: Intelligent Computer Communication and Processing (ICCP), 2016 IEEE 12th International Conference On. Washington, DC: IEEE; 2016. p. 317-20

11. Gabrys R, Yaakobi E, Milenkovic O. Codes in the damerau distance for deletion and adjacent transposition correction. IEEE Trans Inf Theory. 2018;64(4):2550-70.

12. Largest Protein Sequence: Titin. https://en.wikipedia.org/wiki/Titin. Accessed 15 Feb 2019.

13. Largest Chromosome. https://en.wikipedia.org/wiki/Chromosome_1. Accessed 115 Feb 2019

14. Levenshtein VI. Binary codes capable of correcting deletions, insertions, and reversals. Sov Phys Dokl. 1966;10(8):707-10.

15. Robinson DJS. An Introduction to Abstract Algebra. Berlin: Walter de Gruyter; 2003, pp. 255-7.

16. Jaro MA. Advances in record-linkage methodology as applied to matching the 1985 census of Tampa, Florida. J Am Stat Assoc. 1989;84(406):414-20.

17. Lowrance R, Wagner RA. An extension of the string-to-string correction problem. J ACM. 1975;22(2):177-83.

18. Zhao C, Sahni S. String correction using the damerau-levenshtein distance. BMC Bioinforma. 20:277. https://doi.org/10.1186/s12859-0192819-0.

19. Wagner RA, Fischer MJ. The string-to-string correction problem. J ACM. 1974;21(1):168-73.

20. Hirschberg DS. A linear space algorithm for computing longest common subsequences. Commun ACM. 1975;18(6):341-3.

21. Perf Tool. https://perf.wiki.kernel.org/index.php/Main_Page. Accessed 15 Feb 2019.

22. Protein Data Bank. http://www.rcsb.org/pdb/home/home.do .Accessed 15 Feb 2019.

23. NCBI Database. http://www.ncbi.nlm.nih.gov/gquery. Accessed 15 Feb 2019.

\section{Publisher's Note}

Springer Nature remains neutral with regard to jurisdictional claims in published maps and institutional affiliations.

Ready to submit your research? Choose BMC and benefit from

- fast, convenient online submission

- thorough peer review by experienced researchers in your field

- rapid publication on acceptance

- support for research data, including large and complex data types

- gold Open Access which fosters wider collaboration and increased citations

- maximum visibility for your research: over $100 \mathrm{M}$ website views per year

At BMC, research is always in progress.

Learn more biomedcentral.com/submissions 